\title{
SVEIRS: A New Epidemic Disease Model with Time Delays and Impulsive Effects
}

\author{
Tongqian Zhang, ${ }^{1}$ Xinzhu Meng, ${ }^{2}$ and Tonghua $\mathrm{Zhang}^{3}$ \\ ${ }^{1}$ College of Mathematics and Systems Science, Shandong University of Science and Technology, Qingdao 266590, China \\ ${ }^{2}$ Shandong University of Science and Technology, Qingdao 266590, China \\ ${ }^{3}$ Department of Mathematics, Swinburne University of Technology, P.O. Box 218, Hawthorn, VIC 3122, Australia
}

Correspondence should be addressed to Tongqian Zhang; zhangtongqian@sdust.edu.cn

Received 5 January 2014; Revised 12 April 2014; Accepted 25 April 2014; Published 26 May 2014

Academic Editor: Zhiming Guo

Copyright (C) 2014 Tongqian Zhang et al. This is an open access article distributed under the Creative Commons Attribution License, which permits unrestricted use, distribution, and reproduction in any medium, provided the original work is properly cited.

\begin{abstract}
We first propose a new epidemic disease model governed by system of impulsive delay differential equations. Then, based on theories for impulsive delay differential equations, we skillfully solve the difficulty in analyzing the global dynamical behavior of the model with pulse vaccination and impulsive population input effects at two different periodic moments. We prove the existence and global attractivity of the "infection-free" periodic solution and also the permanence of the model. We then carry out numerical simulations to illustrate our theoretical results, showing us that time delay, pulse vaccination, and pulse population input can exert a significant influence on the dynamics of the system which confirms the availability of pulse vaccination strategy for the practical epidemic prevention. Moreover, it is worth pointing out that we obtained an epidemic control strategy for controlling the number of population input.
\end{abstract}

\section{Introduction}

In epidemic modeling, susceptible-infectious-recovered type of models is well known [1-18] although such models very often ignore the incubation period in the development of mathematical models for some diseases. However, recent research shows for certain diseases, such as smallpox, rabies, BSE, and some skin diseases, the incubation period has significant effect on the epidemic dynamics so that it is nonnegligible. The incubation period varies greatly from a couple of days (e.g., H1N1 outbreaking worldwide has generally an incubation period of one to seven days) to several years (e.g., AIDS virus sometimes can be several years). When taking the incubation period into account in the development of models, we reach SEIR model, which is short for susceptible, exposed, infectious, and recovered [19-30]. And some researchers used time delay to describe the incubation period; for example, Cooke [31], Beretta and Takeuchi [4], Takeuchi et al. [32], and Ma et al. [5] studied a SIR model with time delay and nonlinear incidence rate $\beta S(t) I(t-\tau)$. Liu et al. [33, 34] used a nonlinear incidence rate $\beta S^{p}(t) I^{q}(t)$, and Meng et al. [35] and Jiang et al. [30], respectively, studied an impulsively vaccinating SIR model with nonlinear incidences $\beta S^{q}(t) I(t-\tau)$ and $\beta S^{q}(t-\tau) I(t-\tau)$, which are better to describe the spread process of diseases than linear one.

In order to prevent infectious diseases, [36, 37] suggested that vaccination to the susceptible population is an important strategy. The traditional vaccinations are applied to each individual, while impulsive ones are to periodically vaccinate people within certain age groups [7-10, 38]. Some diseases may have a vaccination period after being cured but may cause losing immunity gradually. In this case, people might be infected again. So it is of great significance to investigate epidemic models with time delay and impulsive effects due to the incubation period and vaccination period [26-29]. For some certain regional systems, the immigrations can be periodic impulsive population input because the immigratory population might be susceptible. Certainly two different impulsive effects for periodic vaccination and population input do not usually happen simultaneously. Therefore, motivated by Jiang et al. [30] and Song et al. [19], we built a new mathematical model: susceptible, vaccinated, exposed, 
infectious, recovered, and susceptible epidemic model with two time delays and two nonlinear incidences with pulse vaccination and a constant periodic population input at two different moments as follows:

$$
\begin{aligned}
& \frac{d S(t)}{d t}=-b S(t)-\beta S^{p}(t) I(t)+\gamma I(t-\omega) e^{-b \omega}, \\
& \frac{d V(t)}{d t}=-\delta \beta V^{q}(t) I(t)-\gamma_{1} V(t)-b V(t), \\
& \frac{d E(t)}{d t}=-b E+\beta S^{p}(t) I(t)+\delta \beta V^{q}(t) I(t) \\
& -\beta e^{-b \tau} S^{p}(t) I(t-\tau)-\delta \beta e^{-b \tau} V^{q}(t) I(t-\tau), \\
& \frac{d I(t)}{d t}=\beta e^{-b \tau} S^{p}(t) I(t-\tau)+\delta \beta e^{-b \tau} V^{q}(t) I(t-\tau) \\
& -(\gamma+b+\alpha) I(t) \\
& \frac{d R(t)}{d t}=\gamma_{1} V(t)+\gamma I(t)-b R(t)-\gamma I(t-\omega) e^{-b \omega}, \\
& t \neq(n+l-1) T, \quad t \neq n T, \\
& \Delta S(t)=-\theta S(t), \quad \Delta V(t)=\theta S(t), \quad \Delta E(t)=0, \\
& \Delta I(t)=0, \quad \Delta R(t)=0, \\
& t=(n+l-1) T, \\
& \Delta S(t)=\mu, \quad \Delta V(t)=0, \quad \Delta E(t)=0, \\
& \Delta I(t)=0, \quad \Delta R(t)=0, \\
& t=n T .
\end{aligned}
$$

Here all parameters of system (1) are nonnegative constants. For the significance of parameters in (1), please see literatures Jiang et al. [30] and Song et al. [19]. Terms $\beta S^{p} I$ and $V^{q} I$ are the nonlinear incidence rates, and in our paper we only discuss the case

$$
1 \leq q \leq p
$$

\section{Preliminaries}

Let $N(t)=S(t)+V(t)+E(t)+I(t)+R(t)$, and then it is easy to see that $N(t)$ satisfies the following:

$$
N^{\prime}(t) \leq b(1-N(t)), \quad \lim _{t \rightarrow \infty} \sup N(t) \leq 1 .
$$

Hence, for time $t$ which is large, we obtain $0 \leq S(t)+$ $V(t)+I(t) \leq 1$. Let $\omega=\max \{\tau, \omega\}$ and $C^{+}=\{\varphi=$ $\left.\left(\varphi_{1}(s), \ldots, \varphi_{5}(s)\right) \in C: \varphi_{i}(0)>0\right\}$; here $\varphi_{i}(s)>0$ is bounded function on interval $[-\oplus, 0]$. Since variable $R(t)$ only appears in the fifth equation, system (1) can be further reduced as

$$
\begin{gathered}
\frac{d S(t)}{d t}=-b S(t)-\beta S^{p}(t) I(t)+\gamma I(t-\omega) e^{-b \omega}, \\
\frac{d V(t)}{d t}=-\delta \beta V^{q}(t) I(t)-\gamma_{1} V(t)-b V(t),
\end{gathered}
$$

$$
\begin{array}{r}
\frac{d I(t)}{d t}=\beta e^{-b \tau} S^{p}(t) I(t-\tau)+\delta \beta e^{-b \tau} V^{q}(t) I(t-\tau) \\
-(\gamma+b+\alpha) I(t), \\
t \neq(n+l-1) T, \quad t \neq n T, \\
\Delta S(t)=-\theta S(t), \quad \Delta V(t)=\theta S(t), \quad \Delta I(t)=0, \\
\quad t=(n+l-1) T, \\
\Delta S(t)=\mu, \quad \Delta I(t)=0, \\
\Delta V(t)=0, \quad t=n T,
\end{array}
$$

with the initial conditions

$$
\left(\varphi_{1}(s), \varphi_{2}(s), \varphi_{4}(s)\right) \in C^{+}, \quad \varphi_{i}(0)>0, i=1,2,4 .
$$

Lemma 1 (see $[39,40])$. For the following impulse differential inequalities

$$
\begin{aligned}
& s^{\prime}(t) \leq(\geq) q(t) s(t)+r(t), \quad t \neq t_{k}, \\
& s\left(t_{k}^{+}\right) \leq(\geq) b_{k} s\left(t_{k}\right)+p_{k}, \quad t=t_{k}, k \in N,
\end{aligned}
$$

where $q(t), r(t) \in C\left(R_{+}, R\right), b_{k} \geq 0$, and $p_{k}$ are constants.

Assume the following:

$\left(A_{0}\right)$ the sequence $\left\{t_{k}\right\}$ satisfies $0 \leq t_{0}<t_{1}<t_{2}<\cdots$, with $\lim _{t \rightarrow \infty} t_{k}=\infty$;

$\left(A_{1}\right) w \in P C^{\prime}\left(R_{+}, R\right)$ and $s(t)$ is left-continuous at $t_{k}, k \in N$.

Then

$$
\begin{aligned}
s(t) \leq & (\geq) s\left(t_{0}\right) \prod_{t_{0}<t_{k}<t} b_{k} \exp \left(\int_{t_{0}}^{t} q(u) d u\right) \\
& +\sum_{t_{0}<t_{k}<t}\left(\prod_{t_{k}<t_{j}<t} b_{j} \exp \left(\int_{t_{k}}^{t} q(u) d u\right)\right) p_{k} \\
& +\int_{t_{0}}^{t} \prod_{u<t_{k}<t} b_{k} \exp \left(\int_{u}^{t} q(\theta) d \theta\right) r(u) d u, \\
& t \geq t_{0} .
\end{aligned}
$$

Lemma 2 (see [41]). For the following delay differential equation

$$
\frac{d z(t)}{d t}=a z(t-\theta)-b z(t)
$$


where $a, b$, and $\theta$ are all positive constants and $z(t)>0$ for $t \in[-\theta, 0]$, then we have

$$
\lim _{t \rightarrow \infty} z(t)= \begin{cases}0, & \text { if } a<b \\ +\infty, & \text { if } a>b\end{cases}
$$

Lemma 3 (see [42]). The following system,

$$
\begin{gathered}
\frac{d x(t)}{d t}=-b x(t), \quad \frac{d y(t)}{d t}=-(a+b) y(t), \\
t \neq n T, \quad t \neq(n+l-1) T, \\
\Delta x(t)=-\theta x(t), \quad \Delta y(t)=\theta x(t), \quad t=(n+l-1) T, \\
\Delta x(t)=\mu, \quad \Delta y(t)=0, \quad t=n T,
\end{gathered}
$$

has a unique positive T-periodic solution:

$$
\begin{aligned}
& x^{*}(t)=\left\{\begin{array}{l}
\frac{\mu \exp (-b(t-(n-1) T))}{1-(1-\theta) \exp (-b T)}, \\
t \in((n-1) T,(n+l-1) T], \\
\frac{\mu(1-\theta) \exp (-b(t-(n-1) T))}{1-(1-\theta) \exp (-b T)}, \\
t \in((n+l-1) T, \leq n T], \\
y^{*}(t)=\frac{\mu \theta \exp (-b l T) \exp (-(a+b)(t-(n+l-1) T))}{(1-\exp (-(a+b) T))(1-(1-\theta) \exp (-b T))}, \\
t \in((n+l-1) T,(n+l) T],
\end{array}\right.
\end{aligned}
$$

and we further have $x(t) \rightarrow x^{*}(t)$ and $y(t) \rightarrow y^{*}(t)$ as $t \rightarrow$ $+\infty$.

\section{The Existence and Global Attractivity of "Infection-Free" Periodic Solution}

3.1. Existence. In this section, we are committed to investigate the existence of "infection-free" periodic solution. In this case, we have

$$
I(t)=0, \quad t \geq 0 .
$$

From systems (4) and (12), we obtain

$$
\begin{gathered}
\frac{d S(t)}{d t}=-b S(t), \quad \frac{d V(t)}{d t}=-\left(\gamma_{1}+b\right) V(t), \\
t \neq(n+l-1) T, \quad t \neq n T, \quad n \in N, \\
\Delta S(t)=-\theta S(t), \quad \Delta V(t)=\theta S(t),
\end{gathered}
$$

$$
\begin{gathered}
t=(n+l-1) T, \quad n \in N, \\
\Delta S(t)=\mu, \quad \Delta V(t)=0, \quad t=n T, n \in N .
\end{gathered}
$$

By Lemma 3, system (13) has a unique positive $T$-periodic solution:

$$
\begin{gathered}
S^{*}(t)=\left\{\begin{array}{l}
\frac{\mu \exp (-b(t-(n-1) T))}{1-(1-\theta) \exp (-b T)}, \\
t \in((n-1) T,(n+l-1) T], \\
\frac{\mu(1-\theta) \exp (-b(t-(n-1) T))}{1-(1-\theta) \exp (-b T)}, \\
t \in((n+l-1) T, \leq n T], \\
V^{*}(t)=\frac{\mu \theta \exp (-b l T) \exp (-(a+b)(t-(n+l-1) T))}{(1-\exp (-(a+b) T))(1-(1-\theta) \exp (-b T))}, \\
t \in((n+l-1) T,(n+l) T] .
\end{array}\right.
\end{gathered}
$$

Furthermore, we can prove that it is the unique globally asymptotically stable positive periodic solution of system (4). We summarize this conclusion in the following lemma.

Lemma 4. The system (4) has an "infection-free" periodic solution $\left(S^{*}(t), V^{*}(t), 0\right)$, for $t \in((n+l-1) T,(n+l) T]$ and $n \in N$; for any solution $(S(t), V(t), I(t))$ of $i$, the following holds true:

$$
S(t) \longrightarrow S^{*}(t), \quad V(t) \longrightarrow V^{*}(t)
$$

ast $\rightarrow \infty$.

This lemma indicates that in between the vaccination the susceptible and vaccinated populations oscillate with period $T$ in synchronization with the periodic pulse vaccination. Next we prove the global attractivity of such solution.

3.2. Global Attractivity. In this section, we will prove our main result on the global attractivity of the infection-free solution. It is stated in the following theorem.

Theorem 5. The system (4) has a unique infection-free periodic solution $\left(S^{*}(t), V^{*}(t), 0\right)$, and when it exists, it is globally attractive if

$$
\mathscr{R}_{1}<1 \text {, }
$$

where

$$
\mathscr{R}_{1}=\beta e^{-b \tau} \frac{\left(A_{1}^{p}+\delta A_{2}^{q}\right)}{\gamma+b+\alpha},
$$

with

$$
\begin{aligned}
& A_{1}=\frac{\gamma e^{-b \omega}}{b}+\frac{\mu e^{b T}}{e^{b T}-1} \\
& A_{2}=\frac{\theta e^{-b l T}}{(1-\theta)\left(1-(1-\theta) e^{-b T}\right)\left(1-e^{-\left(\gamma_{1}+b\right) T}\right)} .
\end{aligned}
$$


Proof. Let $(S(t), V(t), I(t))$ be a solution of (4) satisfied initial condition (5). Since $\mathscr{R}_{1}<1$, one can choose an $\varepsilon>0$ small enough such that

$$
\beta e^{-b \tau}\left(\left(\Delta_{1}\right)^{p}+\delta\left(\Delta_{2}\right)^{q}\right)-(\gamma+b+\alpha)<0,
$$

where

$$
\begin{aligned}
& \Delta_{1}=\frac{\gamma e^{-b \omega}}{b}+\frac{\mu e^{b T}}{e^{b T}-1}+\varepsilon \\
& \Delta_{2}=\frac{\theta e^{-b l T}}{(1-\theta)\left(1-(1-\theta) e^{-b T}\right)\left(1-e^{-\left(\gamma_{1}+b\right) T}\right)}+\varepsilon .
\end{aligned}
$$

For $n>n_{1}$, we have

$$
\begin{gathered}
\frac{d S(t)}{d t} \leq b-b S(t)+\gamma e^{-b \omega}, \\
t \neq(n+l-1) T, \quad t \neq n T, \quad n \in N, \\
\Delta S(t)=-\theta S(t), \quad t=(n+l-1) T, n \in N, \\
\Delta S(t)=\mu, \quad t=n T, n \in N .
\end{gathered}
$$

By impulsive differential inequality Lemma 1, we have

$$
\begin{aligned}
S(t) \leq & S\left(n_{1} T^{+}\right) \prod_{n_{1} T^{+}<n T<t} \exp \left(\int_{n_{1} T}^{t}(-b) d s\right) \\
& +\sum_{n_{1} T<n T<t}\left(\prod_{n T<t_{j}<t} \exp \left(\int_{n T}^{t}(-b) d s\right)\right) \mu \\
& +\int_{n_{1} T}^{t} \prod_{s<n T<t} \exp \left(\int_{s}^{t}(-b) d \theta\right) \gamma e^{-b \omega} d s \\
= & S_{1}+S_{2}+S_{3},
\end{aligned}
$$

where

$$
\begin{aligned}
S_{1} & =S\left(n_{1} T^{+}\right) \prod_{n_{1} T^{+}<n T<t} \exp \left(\int_{n_{1}}^{t / T}(-b) d T \xi\right) \\
& =S\left(n_{1} T^{+}\right) e^{-b\left(t-n_{1} T\right)}, \\
S_{2} & =\sum_{n_{1} T<n T<t}\left(\prod_{n T<t_{j}<t} \exp \left(\int_{n T}^{t}(-b) d s\right)\right) \mu \\
& =\sum_{n_{1} T<n T<t}\left(\mu e^{-b(t-n T)}\right)
\end{aligned}
$$

$$
\begin{aligned}
& =\mu \frac{e^{-b\left(t-n_{1}\right) T}-e^{-b\left(t-\left(n+n_{1}\right)\right) T}}{1-e^{b T}}, \\
S_{3} & =\int_{n_{1} T}^{t} \prod_{s<n T<t} \exp \left(\int_{s}^{t}(-b) d \theta\right) \gamma e^{-b \omega} d s \\
& =\gamma e^{-b \omega} \int_{n_{1} T}^{t} \prod_{s<n T<t} \exp \left(\int_{s}^{t}(-b) d \theta\right) d s
\end{aligned}
$$$$
=\frac{\gamma e^{-b \omega} e^{-b t}}{b} \int_{n_{1} T}^{t} \prod_{s<n T<t} e^{b s} d(b s)
$$$$
=\frac{\gamma e^{-b \omega} e^{-b t}}{b}\left(e^{b t}-e^{b n_{1} T}\right) \text {. }
$$

Thus

$$
\begin{aligned}
S(t) \leq & S_{1}+S_{2}+S_{3} \\
= & S\left(n_{1} T^{+}\right) e^{-b\left(t-n_{1} T\right)} \\
& +\mu \frac{e^{-b\left(t-n_{1}\right) T}-e^{-b\left(t-\left(n+n_{1}\right)\right) T}}{1-e^{b T}} \\
& +\frac{\gamma e^{-b \omega} e^{-b t}}{b}\left(e^{b t}-e^{b n_{1} T}\right) \\
\leq & e^{-b t} S\left(n_{1} T^{+}\right) e^{n_{1} b T}+\frac{\mu e^{-b\left(t-n_{1} T\right)}}{1-e^{b T}} \\
& +\frac{\gamma e^{-b \omega}}{b} e^{-b\left(t-n_{1} T\right)}+\frac{\gamma e^{-b \omega}}{b}+\frac{\mu e^{b T}}{e^{b T}-1},
\end{aligned}
$$

and then we have

$$
\lim _{t \rightarrow \infty} \sup S(t)<\frac{\gamma e^{-b \omega}}{b}+\frac{\mu e^{b T}}{e^{b T}-1} .
$$

Thus there exists a positive integer $n_{2}>n_{1}$ and constant $\varepsilon>0$ small enough such that, for all $t>n_{2} T$,

$$
S(t) \leq \frac{\gamma e^{-b \omega}}{b}+\frac{\mu e^{b T}}{e^{b T}-1}+\varepsilon=\Delta_{1} .
$$

For $n>n_{1}$, system (4) yields

$$
\begin{gathered}
\frac{d V(t)}{d t} \leq-\left(\gamma_{1}+b\right) V(t), \quad t \neq(n+l-1) T, n \in N, \\
\Delta V(t)=\theta S(t), \quad t=(n+l-1) T, n \in N .
\end{gathered}
$$

We obtain the following comparison impulsive differential system:

$$
\begin{gathered}
\frac{d x(t)}{d t}=-\left(\gamma_{1}+b\right) x(t), \quad t \neq(n+l-1) T, n \in N, \\
\Delta x(t)=\theta S(t), \quad t=(n+l-1) T, n \in N .
\end{gathered}
$$


By Lemma 3, the system has a periodic solution given by

$$
\begin{array}{r}
x^{*}(t)=\frac{\theta e^{-b l T} e^{-\left(\gamma_{1}+b\right)(t-(n+l-1) T)}}{(1-\theta)\left(1-(1-\theta) e^{-b T}\right)\left(1-e^{-\left(\gamma_{1}+b\right) T}\right)}, \\
t \in((n+l-1) T,(n+l) T], \\
x\left(0^{+}\right)=\frac{\theta e^{-b l T}}{(1-\theta)\left(1-(1-\theta) e^{-b T}\right)\left(1-e^{-\left(\gamma_{1}+b\right) T}\right)},
\end{array}
$$

which is globally asymptotically stable.

Now, assume that $x(t)$ is the solution of system (28) with initial value $x\left(0^{+}\right)=V_{0}$. Then by Lemma 1 , we know there exists a positive integer $n$ such that

$$
V(t)<x(t)<x^{*}(t)+\varepsilon, \quad t \in(n T,(n+1) T] .
$$

Hence,

$$
\begin{aligned}
V(t) & <x(t)<x^{*}(t)+\varepsilon \\
& <\frac{\theta e^{-b l T}}{(1-\theta)\left(1-(1-\theta) e^{-b T}\right)\left(1-e^{-\left(\gamma_{1}+b\right) T}\right)}+\varepsilon_{0} \\
& =\Delta_{2} .
\end{aligned}
$$

From (27), (31), and the third equation in (4), for $t>n_{2} T+\tau$ we have

$$
\frac{d I(t)}{d t} \leq \beta e^{-b \tau}\left(\Delta_{1}^{p}+\delta \Delta_{2}^{q}\right) I(t-\tau)-(\gamma+b+\alpha) I(t) .
$$

Consider the comparison equation:

$$
\frac{d y(t)}{d t} \leq \beta e^{-b \tau}\left(\Delta_{1}^{p}+\delta \Delta_{2}^{q}\right) y(t-\tau)-(\gamma+b+\alpha) y(t) .
$$

From (19), we have

$$
\beta e^{-b \tau}\left(\Delta_{1}^{p}+\delta \Delta_{2}^{q}\right)-(\gamma+b+\alpha)<0 .
$$

According to Lemma 2, we then obtain

$$
\lim _{t \rightarrow \infty} y(t)=0
$$

Notice the fact that $I(s)=y(s)=\phi_{3}(s)>0$ for all $s \in[-\tau, 0]$ and $I(t) \geq 0$, and the comparison theorem implies $I(t) \rightarrow 0$ as $t \rightarrow \infty$. Without loss of generality, we may assume that $0<I(t)<\varepsilon_{1}$ for all $t \geq 0$. By using the first and second equations in (4), we reach

$$
\begin{gathered}
\frac{d S(t)}{d t} \geq-b S(t)-\beta \varepsilon_{1} S^{p}(t), \\
\frac{d V(t)}{d t} \geq-\delta \beta \varepsilon_{1} V^{q}(t)-\gamma_{1} V(t)-b V(t), \\
t \neq(n+l-1) T, \quad t \neq n T, \quad n \in N,
\end{gathered}
$$

$$
\begin{gathered}
\Delta S(t)=-\theta S(t), \quad \Delta V(t)=\theta S(t), \\
t=(n+l-1) T, \quad n \in N, \\
\Delta S(t)=\mu, \quad \Delta V(t)=0, \quad t=n T, n \in N .
\end{gathered}
$$

For $1<q<p$, we have

$$
\begin{gathered}
\frac{d S(t)}{d t} \geq-b S(t)-\beta \varepsilon_{1} S(t), \\
\frac{d V(t)}{d t} \geq-\delta \beta \varepsilon_{1} V(t)-\gamma_{1} V(t)-b V(t), \\
t \neq(n+l-1) T, \quad t \neq n T, \quad n \in N, \\
\Delta S(t)=-\theta S(t), \quad \Delta V(t)=\theta S(t), \\
\quad t=(n+l-1) T, \quad n \in N, \\
\Delta S(t)=\mu, \quad \Delta V(t)=0, \quad t=n T, n \in N
\end{gathered}
$$

considering the following system:

$$
\begin{gathered}
\frac{d f(t)(t)}{d t}=-b f(t)-\beta \varepsilon_{1} f(t), \\
\frac{d g(t)}{d t}=-\delta \beta \varepsilon_{1} g(t)-\gamma_{1} g(t)-b g(t), \\
t \neq(n+l-1) T, \quad t \neq n T, \quad n \in N, \\
\Delta f(t)=-\theta f(t), \quad \Delta g(t)=\theta f(t), \\
t=(n+l-1) T, \quad n \in N, \\
\Delta f(t)=\mu, \quad \Delta g(t)=0, \quad t=n T, n \in N .
\end{gathered}
$$

We obtain

$$
\tilde{f}(t)=\left\{\begin{array}{c}
\frac{\left(b+\beta \varepsilon_{1}\right) \mu}{\left(b+\beta \varepsilon_{1}\right)\left(1-(1-\theta) e^{-\left(b+\beta \varepsilon_{1}\right) T}\right)} \\
\times e^{-\left(b+\beta \varepsilon_{1}\right)(t-(n-1) T)}, \\
t \in((n-1) T,(n+l-1) T], \\
\frac{\left(b+\beta \varepsilon_{1}\right) \mu(1-\theta) e^{-\left(b+\beta \varepsilon_{1}\right) l T}}{\left(b+\beta \varepsilon_{1}\right)\left(1-(1-\theta) e^{-\left(b+\beta \varepsilon_{1}\right) T}\right)} \\
\times e^{-\left(b+\beta \varepsilon_{1}\right)(t-(n+l-1) T)}, \\
t \in((n+l-1) T, n T],
\end{array}\right.
$$




$$
\begin{aligned}
& \tilde{g}(t)=\left(\left(\theta\left(b+\beta \varepsilon_{1}\right) \mu e^{-\left(b+\beta \varepsilon_{1}\right) l T}-\beta \varepsilon_{1} \theta\left(1-e^{-\left(b+\beta \varepsilon_{1}\right) T}\right)\right)\right. \\
&\left.\times e^{-\left(\gamma_{1}+b+\delta \beta \varepsilon_{1}\right)(t-(n+l-1) T)}\right) \\
& \times\left(\left(b+\beta \varepsilon_{1}\right)\left(1-(1-\theta) e^{-\left(b+\beta \varepsilon_{1}\right) T}\right)\right. \\
&\left.\times\left(1-e^{-\left(\gamma_{1}+b\right) T}\right)\right)^{-1}, \\
& t \in((n+l-1) T,(n+l) T] .
\end{aligned}
$$

Now by using comparison theorem of impulsive equations, for any $\varepsilon_{2}>0$ there exists a $T_{1}>0$ such that

$$
\begin{aligned}
S(t) & >\tilde{f}(t)-\varepsilon_{2}, \\
V(t) & >\tilde{g}(t)-\varepsilon_{2},
\end{aligned}
$$

for $t>T_{1}$. On the other side, from the first and second equations of (4), we have

$$
\begin{gathered}
\frac{d S(t)}{d t} \leq-b S(t)+\gamma \varepsilon_{1} e^{-b \omega}, \\
\frac{d V(t)}{d t} \leq-\gamma_{1} V(t)-b V(t), \\
t \neq(n+l-1) T, \quad t \neq n T, \quad n \in N, \\
\Delta S(t)=-\theta S(t), \quad \Delta V(t)=\theta S(t), \\
t=(n+l-1) T, \quad n \in N, \\
\Delta S(t)=b, \quad \Delta V(t)=0, \quad t=n T, n \in N .
\end{gathered}
$$

Then we have $S(t) \leq \tilde{h}(t), V(t) \leq \tilde{g}(t)$ and $\tilde{h}(t) \rightarrow S^{*}(t)$, $\tilde{g}(t) \rightarrow V^{*}(t)$, as $\varepsilon_{1} \rightarrow 0$, where $(\widetilde{h}(t), \tilde{g}(t))$ is a unique positive periodic solution of

$$
\begin{gathered}
\frac{d h(t)}{d t}=-b h(t)+\gamma \varepsilon_{1} e^{-b \omega}, \\
\frac{d g(t)}{d t}=-\gamma_{1} g(t)-b g(t), \\
t \neq(n+l-1) T, \quad t \neq n T, \quad n \in N, \\
\Delta h(t)=-\theta h(t), \quad \Delta g(t)=\theta h(t), \\
t=(n+l-1) T, \quad n \in N, \\
\Delta h(t)=\mu, \quad \Delta g(t)=0, \quad t=n T, n \in N,
\end{gathered}
$$

from which we have that, for $n T<t \leq(n+1) T$,

$$
\tilde{h}(t)=\left\{\begin{array}{l}
\frac{\gamma \varepsilon_{1} e^{-b \omega} \theta e^{-b(1-l) T}+b \mu}{b\left(1-(1-\theta) e^{-b T}\right)} \\
\times e^{-b(t-(n-1) T)}-\frac{\gamma \varepsilon_{1} e^{-b \omega}}{b}, \\
\quad(n-1) T<t \leq(n+l-1) T, \\
\frac{b \mu(1-\theta) e^{-b l T}+\gamma \varepsilon_{1} e^{-b \omega} \theta}{b\left(1-(1-\theta) e^{-b T}\right)} \\
\times e^{-b(t-(n+l-1) T)}-\frac{\gamma \varepsilon_{1} e^{-b \omega}}{b}, \\
(n+l-1) T<t \leq n T, \\
\tilde{g}(t)=\frac{\left(\theta b \mu e^{-b l T}-\gamma \varepsilon_{1} e^{-b \omega} \theta\left(1-e^{-b T}\right)\right) e^{-\left(\gamma_{1}+b\right)(t-(n+l-1) T)}}{b\left(1-(1-\theta) e^{-b T}\right)\left(1-e^{-\left(\gamma_{1}+b\right) T}\right)},
\end{array}\right.
$$$$
(n+l-1) T<t \leq(n+l) T .
$$

Applying the comparison theorem again, for any $\varepsilon_{2}>0$, there exists a $T_{2}>0$ such that

$$
\begin{aligned}
S(t) & <\tilde{h}(t)-\varepsilon_{2}, \\
V(t) & <\tilde{g}(t)-\varepsilon_{2},
\end{aligned}
$$

for $t>T_{2}$. Let $\varepsilon_{1} \rightarrow 0$, and then from (40) and (44) we have

$$
\begin{gathered}
S^{*}(t)-\varepsilon_{2}<S(t)<S^{*}(t)-\varepsilon_{2}, \\
V^{*}(t)-\varepsilon_{2}<V(t)<V^{*}(t)-\varepsilon_{2},
\end{gathered}
$$

for $t$ large enough, which implies $S(t) \rightarrow S^{*}(t), V(t) \rightarrow$ $V^{*}(t)$ as $t \rightarrow \infty$. This completes the proof.

Corollary 6. If $\tau>\tau^{*}$ or $\mu<\mu^{*}$, then the infection-free periodic solution $\left(S^{*}(t), V^{*}(t), 0\right)$ is globally attractive, where the critical values are given below:

$$
\begin{aligned}
\tau^{*}= & \frac{1}{b} \ln \frac{\beta\left(A_{1}^{p}+\delta A_{2}^{q}\right)}{\gamma+b+\alpha}, \\
\mu^{*}= & \left(1-e^{-b T}\right) \\
& \times\left(\sqrt[p]{\frac{\gamma+b+\alpha}{\beta} e^{b \tau}-\delta A_{2}^{q}}-\frac{\gamma e^{-b \omega}}{b}-\frac{\mu e^{b T}}{e^{b T}-1}\right) .
\end{aligned}
$$

\section{Permanence}

In this section, we discuss the permanence of the infectious population. First, we introduce the following definition. 
Definition 7. System (4) is said to be permanent if there exist positive constants $m_{i}, M_{i}, i=1,2,3$ (independent of initial value), and a finite time $T_{0}$, which may depend on the initial condition, such that every positive solution $(S(t), V(t), I(t))$ with initial condition (5) satisfies $m_{1} \leq S(t) \leq M_{1}, m_{2} \leq$ $I(t) \leq M_{2}, m_{3} \leq V(t) \leq M_{3}$ for all $t>T_{0}$.

$$
\begin{aligned}
& \text { Let } \\
& S^{*}=\sqrt[p]{\frac{\gamma+b+\alpha}{\beta e^{-b \tau}}}, \\
& V^{*}=\sqrt[q]{\frac{\gamma_{1}+b}{\delta \beta e^{-b \tau}}}, \\
& m^{*}=\frac{(1 / T) \ln \left(\left(\Re_{1}\left(e^{b T}-1+\theta\right)+1-\theta+e^{b T}\right) / 2\right)-b}{\beta}, \\
& \mathfrak{R}_{1}=\sqrt[p]{\frac{\beta e^{-b \tau}}{\gamma+b+\alpha}}\left(\frac{\mu(1-\theta) e^{-b T}}{1-(1-\theta) e^{-b T}}\right), \\
& \mathfrak{R}_{2}=\sqrt[q]{\frac{\beta e^{-b \tau}}{\gamma_{1}+b} \overline{\left(1-e^{-\left(\gamma_{1}+b\right) T}\right)\left(1-(1-\theta) e^{-b T}\right)},} \\
& \mathscr{R}_{2}=\min \left\{\mathfrak{R}_{1}, \mathfrak{R}_{2}\right\} .
\end{aligned}
$$

Then we have our main result of this section.

Theorem 8. Let $1 \leq q \leq p$, if $\mathscr{R}_{2}>1$, and then there exists a positive constant $\eta$ small enough such that

$$
I(t) \geq \min \left\{\frac{\eta m^{*}}{2}, \eta m^{*} e^{-(\gamma+b+\alpha) \omega}\right\}=m_{1}
$$

with t large enough.

Proof. As before, we suppose that $X(t)=(S(t), V(t), I(t))$ is a positive solution of system (4) with initial condition (5). Then for $t \geq 0$, we construct a function as follows:

$$
\begin{aligned}
U(t)= & I(t)+V(t)+\beta e^{-b \tau}\left(S^{*}\right)^{p} \\
& \times \int_{t-\tau}^{t} I(\varrho) d \varrho+\delta \beta e^{-b \tau}\left(V^{*}\right)^{q} \int_{t-\tau}^{t} V(\varrho) d \varrho .
\end{aligned}
$$

And then differentiating $U(t)$ along the trajectory of (4) yields

$$
\begin{aligned}
\dot{U}(t)= & \dot{I}(t)+\dot{V}(t)+\beta e^{-b \tau}\left(S^{*}\right)^{p} I(t) \\
& -\beta e^{-b \tau}\left(S^{*}\right)^{p} I(t-\tau)+\delta \beta e^{-b \tau}\left(V^{*}\right)^{q} I(t)
\end{aligned}
$$

$$
\begin{aligned}
& -\delta \beta e^{-b \tau}\left(V^{*}\right)^{q} I(t-\tau) \\
= & \beta e^{-b \tau}\left(S^{p}(t)-\left(S^{*}\right)^{p}\right) I(t-\tau) \\
& +\beta e^{-b \tau}\left(V^{q}(t)-\left(V^{*}\right)^{q}\right) I(t-\tau) \\
& +\left(\beta e^{-b \tau}\left(S^{*}\right)^{p}-(\gamma+b+\alpha)\right) I(t) \\
& +\left(\delta \beta e^{-b \tau}\left(V^{*}\right)^{q}-\left(\gamma_{1}+b\right)\right) I(t) \\
= & \beta e^{-b \tau}\left(S^{p}(t)-\left(S^{*}\right)^{p}\right) I(t-\tau) \\
& +\beta e^{-b \tau}\left(V^{q}(t)-\left(V^{*}\right)^{q}\right) I(t-\tau) \\
= & \beta e^{-b \tau}\left(S^{p-1}(t)+S^{p-2}(t) S^{*}+\cdots\right. \\
& \left.\quad+S(t)\left(S^{*}\right)^{p-2}+\left(S^{*}\right)^{p-1}\right) \\
& +\left(S(t)-S^{*}\right) I(t-\tau) \\
& +\delta \beta e^{-b \tau}\left(V^{q-1}(t)+V^{q-2}(t) V^{*}+\cdots\right. \\
& \left.\quad+\left(V(t)-V^{*}\right) I(t-\tau)\left(V^{*}\right)^{q-2}+\left(V^{*}\right)^{q-1}\right)
\end{aligned}
$$

for $t \geq 0$. Let

$$
\begin{aligned}
m^{*} & =\frac{(1 / T) \ln \left(\left(\Re_{1}\left(e^{b T}-1+\theta\right)+1-\theta+e^{b T}\right) / 2\right)-b}{\beta}, \\
S^{*} & =\sqrt[p]{\frac{\gamma+b+\alpha}{\beta e^{-b \tau}}} .
\end{aligned}
$$

Since $\mathscr{R}_{2}>1$, we get $\Re_{1}>1, \Re_{2}>1$. Then we have $m^{*}>0$. And from $\Re_{1}>1$, we can get

$$
\sqrt[p]{\frac{\beta e^{-b \tau}}{\gamma+b+\alpha}}\left(\frac{\mu(1-\theta) e^{-b T}}{1-(1-\theta) e^{-b T}}\right)>1 .
$$

Thus, we have

$$
\frac{\mu(1-\theta) e^{-b T}}{1-(1-\theta) e^{-b T}}>\sqrt[p]{\frac{\gamma+b+\alpha}{\beta e^{-b \tau}}}=S^{*} .
$$


Form $\mathfrak{R}_{2}>1$, we have

$$
\sqrt[q]{\frac{\beta e^{-b \tau}}{\gamma_{1}+b}} \frac{\mu \theta e^{-\left(\gamma_{1}+b+b l\right) T}}{\left(1-e^{-\left(\gamma_{1}+b\right) T}\right)\left(1-(1-\theta) e^{-b T}\right)}>1 ;
$$

that is,

$$
\frac{\mu \theta e^{-\left(\gamma_{1}+b+b l\right) T}}{\left(1-e^{-\left(\gamma_{1}+b\right) T}\right)\left(1-(1-\theta) e^{-b T}\right)}>\sqrt[q]{\frac{\gamma_{1}+b}{\beta e^{-b \tau}}}=V^{*} .
$$

We can take $\eta$ small enough such that

$$
\begin{gathered}
\frac{\mu(1-\theta) e^{-\left(\beta \eta m^{*}+b\right) T}}{1-(1-\theta) e^{-\left(\beta \eta m^{*}+b\right) T}}>S^{*}, \\
\frac{\mu \theta e^{-\left(\beta(\delta+l) \eta m^{*}+\gamma_{1}+b+b l\right) T}}{\left(1-e^{-\left(\delta \beta \eta m^{*}+\gamma_{1}+b\right) T}\right)\left(1-(1-\theta) e^{-\left(\beta \eta m^{*}+b\right) T}\right)}>V^{*} .
\end{gathered}
$$

Thus we can choose $\varepsilon_{1}, \varepsilon_{2}>0$ to be small enough such that

$$
\begin{aligned}
S^{*}< & \frac{\mu(1-\theta) e^{-\left(\beta \eta m^{*}+b\right) T}}{1-(1-\theta) e^{-\left(\beta \eta m^{*}+b\right) T}}-\varepsilon_{1} \equiv S_{\Delta}, \\
V^{*}< & \frac{\mu \theta e^{-\left(\beta(\delta+l) \eta m^{*}+\gamma_{1}+b+b l\right) T}}{\left(1-e^{-\left(\delta \beta \eta m^{*}+\gamma_{1}+b\right) T}\right)\left(1-(1-\theta) e^{-\left(\beta \eta m^{*}+b\right) T}\right)} \\
& \quad-\varepsilon_{2} \equiv V_{\Delta} .
\end{aligned}
$$

Then we claim that there exists an $m_{2}>0$ such that $I(t)>$ $m_{2}$ for $t$ is large enough. We next prove this claim in two steps.

Step I. For any positive constant $t_{0}$, that $I(t) \leq \eta m^{*}$ for all $t \geq t_{0}$ is not true.

Otherwise, there is a positive constant $t_{0}$, such that $I(t) \leq$ $\eta m^{*}$ for all $t \geq t_{0}$. First, if $I(t)<\eta m^{*}$ for all $t \geq t_{0}$, it follows from the first, fourth, and fifth equations of (4) that, for $t \geq t_{0}$,

$$
\begin{aligned}
& \frac{d S(t)}{d t} \geq-\left(\beta \eta m^{*}+b\right) S(t), \quad t \neq(n+l-1) T, t \neq n T, \\
& \Delta S(t)=-\theta S(t), \quad t=(n+l-1) T, \\
& \Delta S(t)=\mu, \quad t=n T .
\end{aligned}
$$

By Lemma 1, there exists $T_{1}>t_{0}+\tau$ so that for $t>T_{1}$

$$
S(t)>\frac{\mu(1-\theta) e^{-\left(\beta \eta m^{*}+b\right) T}}{1-(1-\theta) e^{-\left(\beta \eta m^{*}+b\right) T}}-\varepsilon \equiv S_{\Delta} .
$$

Similarly, from the second and the fourth equations of (4), we have

$$
\begin{aligned}
& \frac{d V(t)}{d t} \geq-\left(\delta \beta \eta m^{*}+\gamma_{1}+b\right) V(t), \quad t \neq(n+l-1) T, \\
& \Delta V(t)=\theta S(t), \quad t=(n+l-1) T,
\end{aligned}
$$

and for $t>T_{1}$,

$$
\begin{aligned}
V(t) \geq & \frac{\mu \theta e^{-\left(\beta(\delta+l) \eta m^{*}+\gamma_{1}+b+b l\right) T}}{\left(1-e^{-\left(\delta \beta \eta m^{*}+\gamma_{1}+b\right) T}\right)\left(1-(1-\theta) e^{-\left(\beta \eta m^{*}+b\right) T}\right)} \\
& -\varepsilon \equiv V_{\Delta} .
\end{aligned}
$$

Then, by (50), for $t \geq T_{1}$,

$$
\begin{aligned}
\dot{U}(t)= & \beta e^{-b \tau}\left(S^{p-1}(t)+S^{p-2}(t) S^{*}+\cdots\right. \\
& \left.+S(t)\left(S^{*}\right)^{p-2}+\left(S^{*}\right)^{p-1}\right) \\
& \times\left(S(t)-S^{*}\right) I(t-\tau) \\
& +\delta \beta e^{-b \tau}\left(V^{q-1}(t)+V^{q-2}(t) V^{*}+\cdots\right. \\
& \left.+V(t)\left(V^{*}\right)^{q-2}+\left(V^{*}\right)^{q-1}\right) \\
& \times\left(V(t)-V^{*}\right) I(t-\tau) \\
> & p \beta e^{-b \tau}\left(S^{*}\right)^{p-1}\left(S_{\Delta}-S^{*}\right) I(t-\tau) \\
& +q \delta \beta e^{-b \tau}\left(V^{*}\right)^{q-1}\left(V_{\Delta}-V^{*}\right) I(t-\tau) .
\end{aligned}
$$

Let

$$
I_{L}=\min _{t \in\left[T_{1}, T_{1}+\tau\right]} I(t)
$$

We can prove that $I(t) \geq I_{L}$ for all $t \geq T_{1}$. Otherwise, there exists a nonnegative constant $T_{2}$ such that $I(t) \geq I_{L}$ for $t \in$ $\left[T_{1}, T_{1}+\tau+T_{2}\right], I\left(T_{1}+\tau+T_{2}\right)=I_{L}$, and $\dot{I}\left(T_{1}+\tau+T_{2}\right) \leq 0$. 


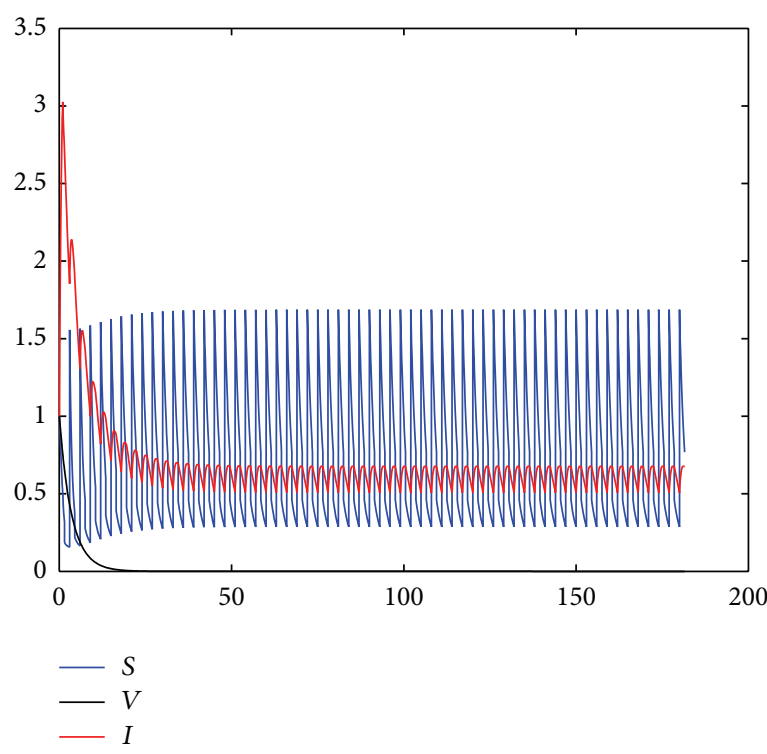

(a) Time series of $S, V$, and $I$. The disease will be permanent

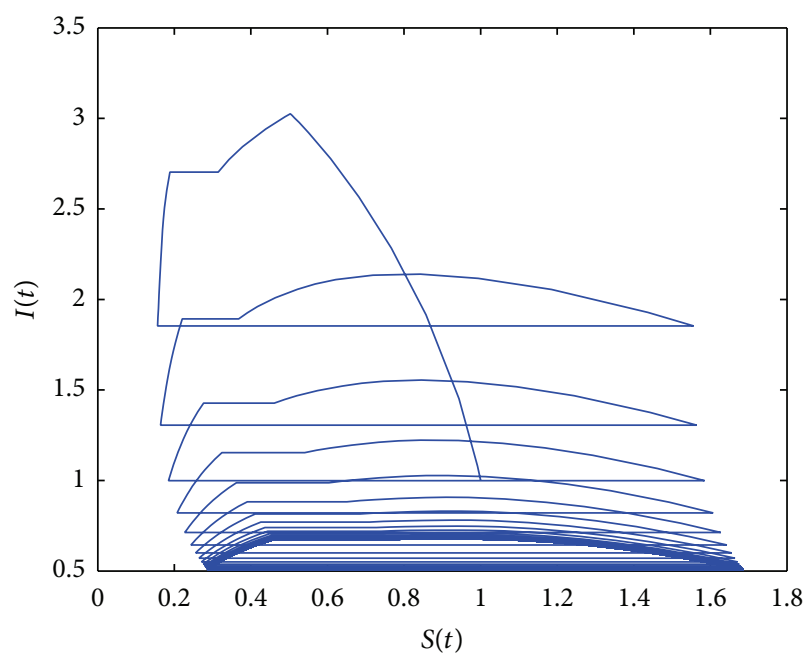

(c) Phase portrait of $S(t), I(t)$ of the system (4)

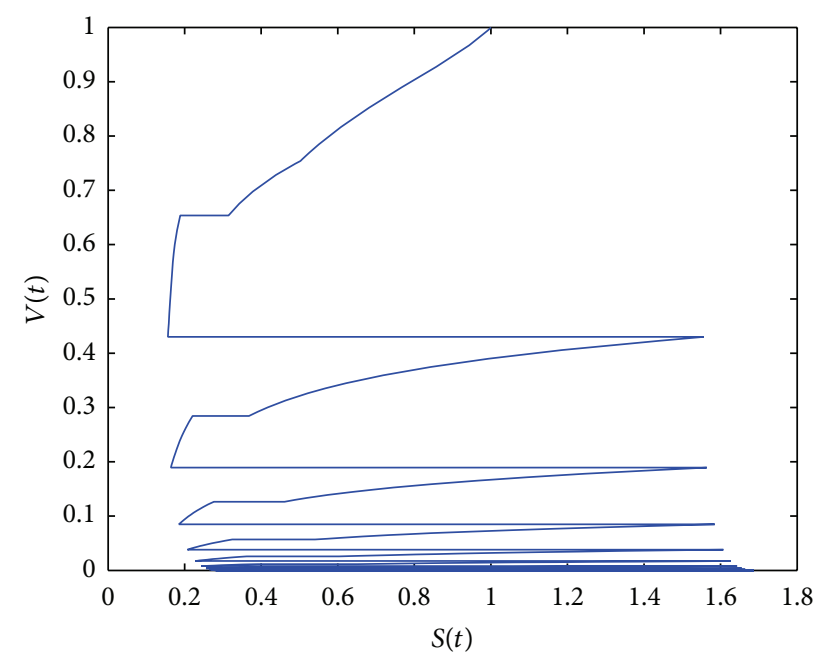

(b) Phase portrait of $S(t), V(t)$ of the system (4)

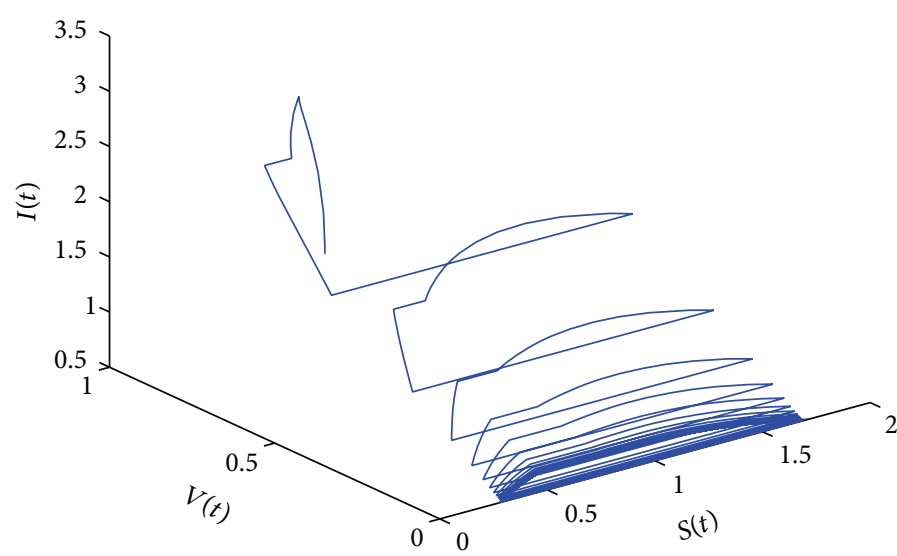

(d) Phase portrait of $S(t), V(t)$, and $I(t)$ of the system (4)

Figure 1: The results of numerical simulation on the threshold values $\mathscr{R}_{2}=2.6155>1$, where $p=1.5, q=1.25$.

Then from the second equation of (4) and (37), we easily see that

$$
\begin{aligned}
\dot{I} & \left(T_{1}+\tau+T_{2}\right) \\
& \geq\left(\beta e^{-b \tau} S^{p}(t)+\delta \beta e^{-b \tau} V^{q}(t)-(\gamma+b+\alpha)\right) I_{L} \\
& =(\gamma+b+\alpha)\left(\frac{\beta e^{-b \tau} S^{p}(t)}{\gamma+b+\alpha}+\frac{\delta \beta e^{-b \tau} V^{q}(t)}{\gamma+b+\alpha}-1\right) I_{L} \\
& >(\gamma+b+\alpha)\left(\left(\frac{S_{\Delta}}{S^{*}}\right)^{p}+\frac{\gamma_{1}+b}{\gamma+b+\alpha}\left(\frac{V_{\Delta}}{V^{*}}\right)^{q}-1\right) I_{L} \\
& >0,
\end{aligned}
$$

which is a contradiction. Hence $I(t) \geq I_{L}>0$ for all $t \geq T_{1}$. Equation (62) implies

$$
\begin{aligned}
\frac{d U(t)}{d t}> & p \beta e^{-b \tau}\left(S^{*}\right)^{p-1}\left(S_{\Delta}-S^{*}\right) I(t-\tau) \\
& +q \delta \beta e^{-b \tau}\left(V^{*}\right)^{q-1}\left(V_{\Delta}-V^{*}\right) I(t-\tau) \\
> & 0 .
\end{aligned}
$$

It then follows that $U(t) \rightarrow+\infty$ as $t \rightarrow+\infty$. This is a contradiction to $U(t) \leq\left(\alpha+\gamma+\gamma_{1}+2 b\right) \tau+2$. Therefore, for any positive constant $t_{0}$, the inequality $I(t)<\eta m^{*}$ cannot hold for all $t \geq t_{0}$.

Step II. From Step I, we only need to consider the followng: (i) $I(t)>\eta m^{*}$ for all $t$ large enough and (ii) $I(t)$ oscillates 


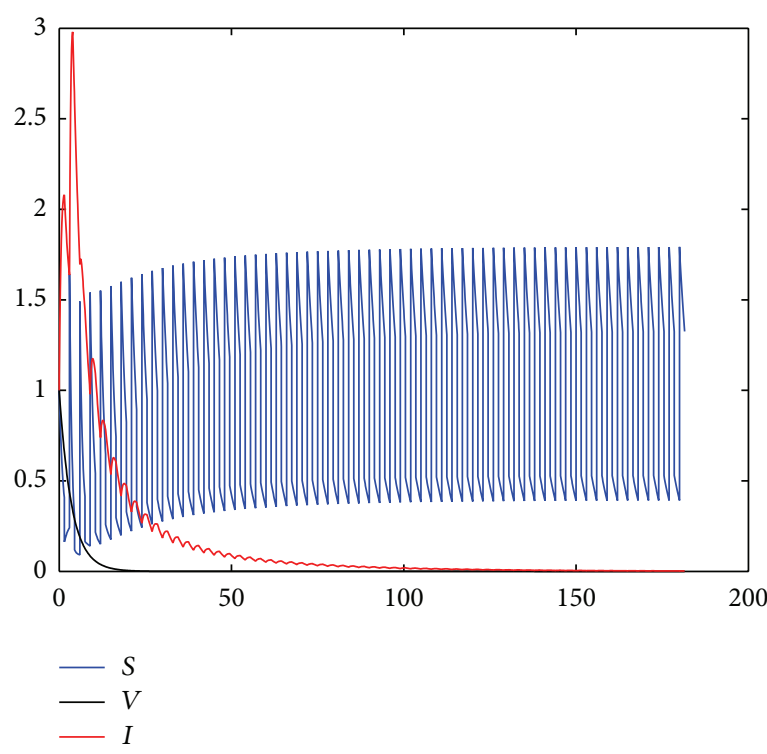

(a) Time series of $S, V$, and $I$. The disease dies out

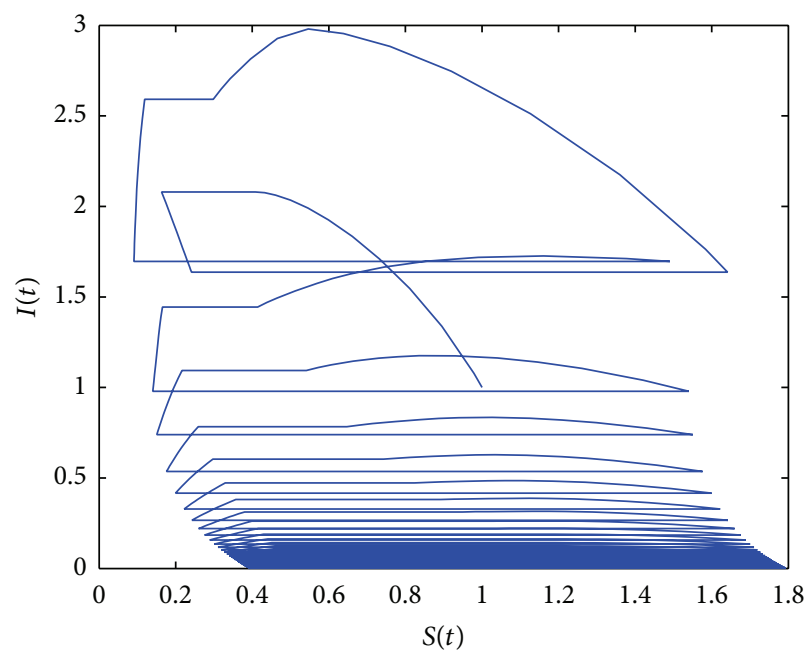

(c) Phase portrait of $S(t), I(t)$ of the system (4)

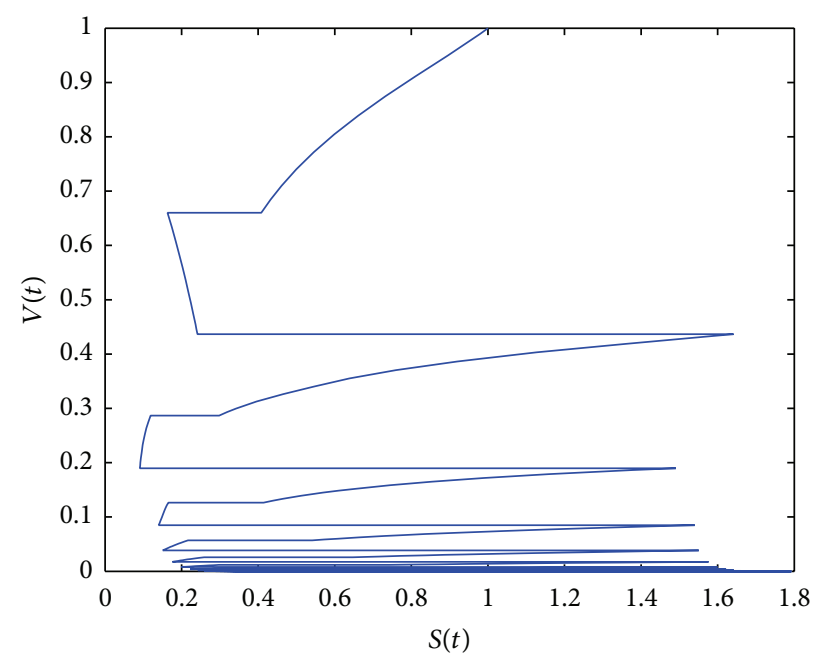

(b) Phase portrait of $S(t), V(t)$ of the system (4)

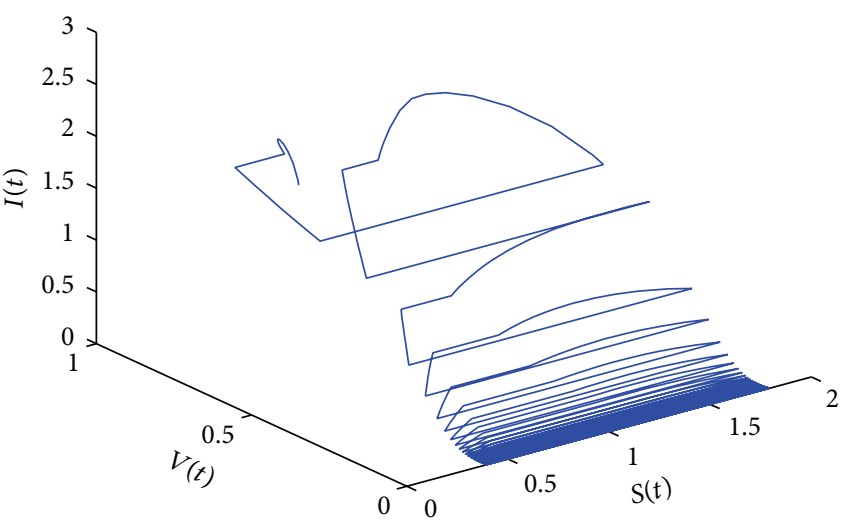

(d) Phase portrait of $S(t), V(t)$, and $I(t)$ of the system (4)

Figure 2: The results of numerical simulation on the threshold values $\mathscr{R}_{1}=0.0339<1$, where $p=1.5, q=1.25$.

about $\eta m^{*}$ for all large $t$. However, Case (i) is obvious in the result of this theorem, so we only need to consider Case (ii), in which we will show that $I(t) \geq m_{1}$ for all large $t$ where

$$
m_{1}=\min \left\{\frac{\eta m^{*}}{2}, \eta m^{*} e^{-(\gamma+b+\alpha) \omega}\right\} .
$$

First, we notice there exist two positive constants $\bar{t}, \varphi$ such that

$$
\begin{aligned}
& I(\bar{t})=I(\bar{t}+\varphi)=I^{*} \\
& I(t)<\eta m^{*}, \quad \text { for } \bar{t}<t<\bar{t}+\varphi .
\end{aligned}
$$

Second, because $I(t)$ is bounded continuous function and $I(t)$ has no pulse, we can get that $I(t)$ is uniformly continuous. Therefore there exists a constant $T_{3}$ (with $0<T_{3}<\omega$ and $T_{3}$ is independent of the choice of $\bar{t}$ ) such that $I(t)>\eta m^{*} / 2$ for all $\bar{t} \leq t \leq \bar{t}+T_{3}$.

If $\varphi \leq T_{3}$, our aim is obtained.

If $T_{3}<\varphi \leq \omega$, from the second equation of (4) we have that $\dot{I}(t) \geq-(\gamma+b+\alpha) I(t)$ for $\bar{t}<t \leq \bar{t}+\varphi$. Then we have $I(t) \geq \eta m^{*} e^{-(\gamma+b+\alpha) \omega}$ for $\bar{t}<t \leq \bar{t}+\varphi \leq \bar{t}+\omega$ since $I(\bar{t})=\eta m^{*}$. It is clear that $I(t) \geq m_{1}$ for $\bar{t}<t \leq \bar{t}+\varphi$.

If $\varphi \geq \omega$, then we have $I(t) \geq m_{2}$ for $\bar{t}<t \leq \bar{t}+\omega$. We then can easily prove $I(t) \geq m_{1}$ for $\bar{t}+\omega \leq t \leq \bar{t}+\varphi$. Since the interval $[\bar{t}, \bar{t}+\varphi]$ is arbitrarily chosen, we know that $I(t) \geq m_{1}$ holds for $t$ large enough. Finally, noticing the choice of $m_{1}$ is independent of the positive solution of (4), we completed our proof. 


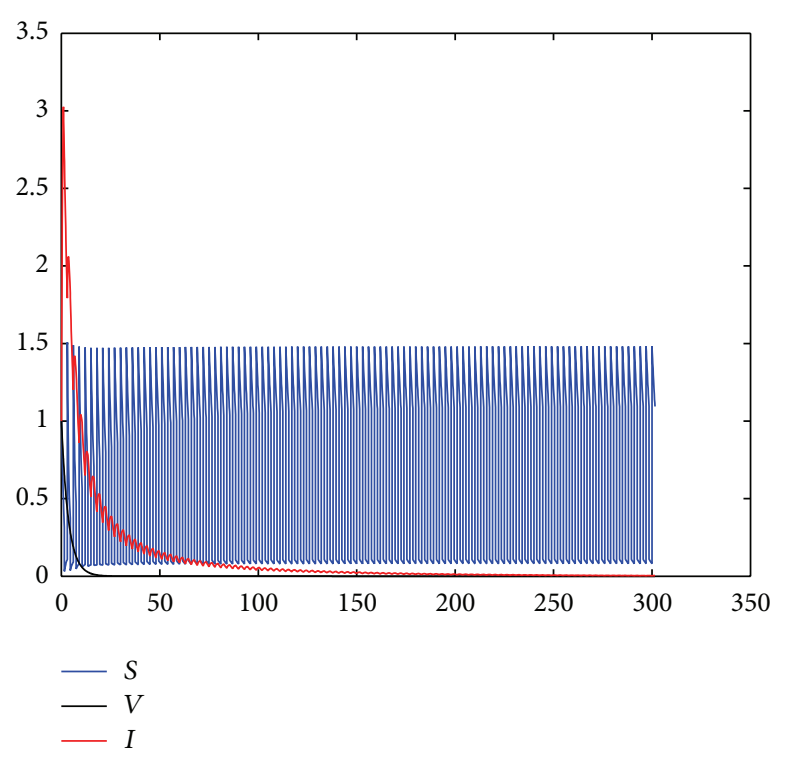

(a) Time series of $S, V$, and $I$. The disease dies out

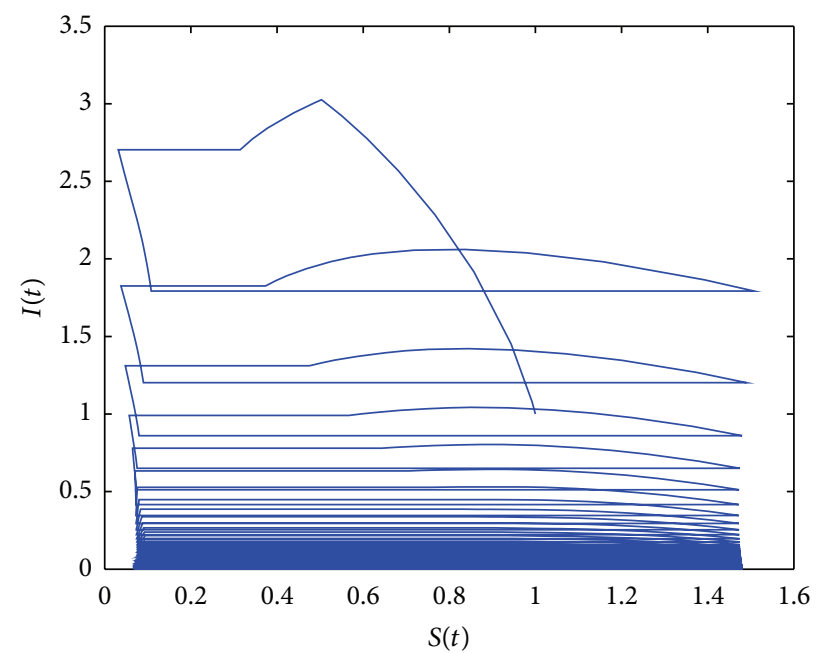

(c) Phase portrait of $S(t), I(t)$ of the system (4)

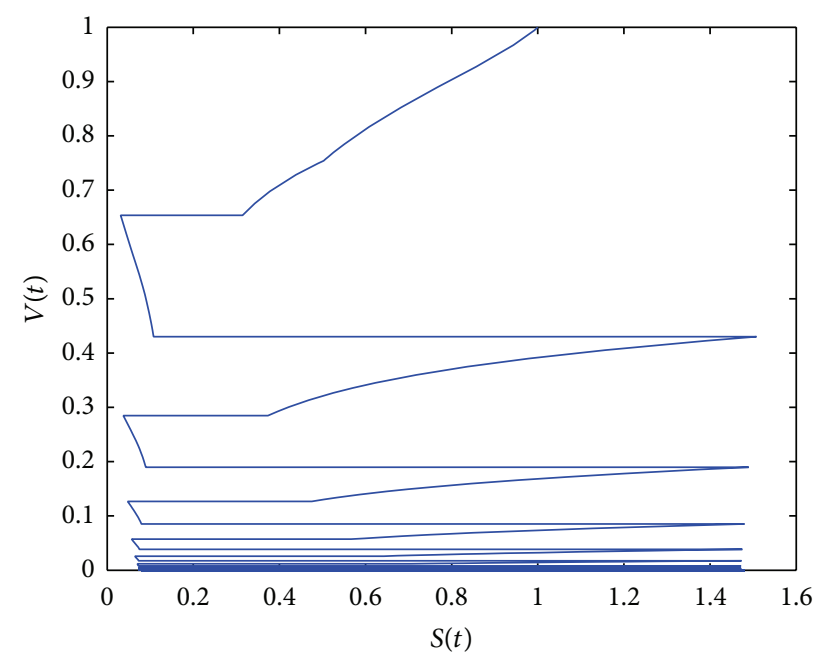

(b) Phase portrait of $S(t), V(t)$ of the system (4)

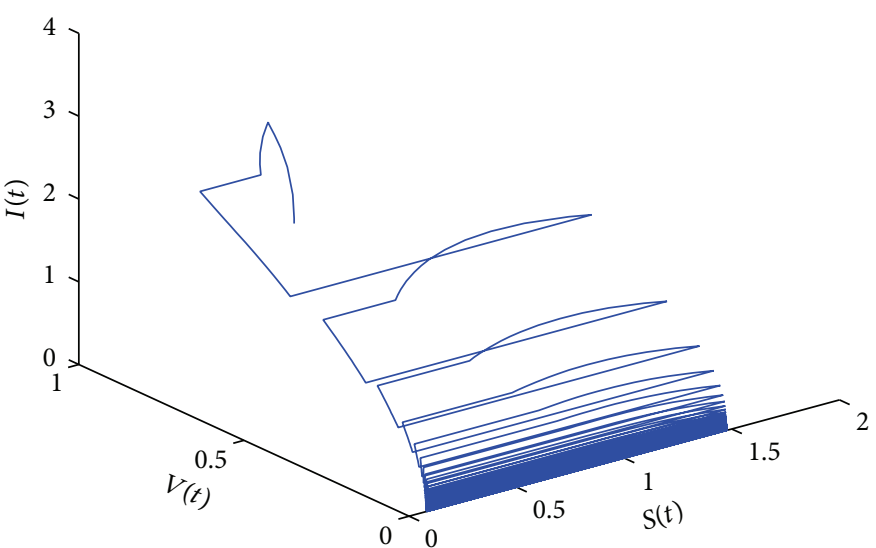

(d) Phase portrait of $S(t), V(t)$, and $I(t)$ of the system (4)

Figure 3: The results of numerical simulation on the threshold values $\mathscr{R}_{1}=0.0449<1$, where $p=1.5, q=1.25$.

Theorem 9. Let $1 \leq q \leq p$, if $\mathscr{R}_{2}>1$, and then system (4) is permanent.

Proof. Suppose that $X(t)=(S(t), V(t), I(t))$ is a positive solution of system (4) with initial conditions (5). Then from system (4), we have

$$
\begin{gathered}
\frac{d S(t)}{d t} \geq-(b+\beta) S(t), \\
\frac{d V(t)}{d t} \geq-\left(\delta \beta+\gamma_{1}+b\right) V(t), \\
t \neq(n+l-1) T, \quad t \neq n T, \quad n \in N, \\
\Delta S(t)=-\theta S(t), \quad \Delta V(t)=\theta S(t),
\end{gathered}
$$

$$
\begin{gathered}
t=(n+l-1) T, \quad n \in N, \\
\Delta S(t)=\mu, \quad \Delta V(t)=0, \quad t=n T, n \in N .
\end{gathered}
$$

As what we did in the proof of Theorem 5, we can prove that there exist $t$ large enough and $\varepsilon>0$ small enough such that

$$
\begin{aligned}
S(t) \geq & \frac{\mu(1-\theta) e^{-(b+\beta) T}}{1-(1-\theta) e^{-(b+\beta) T}}-\varepsilon=m_{3}, \\
V(t) \geq & \frac{\mu \theta e^{-\left(b+\gamma_{1}+\delta \beta+(b+\beta) l\right) T}}{\left(1-(1-\theta) e^{-(b+\beta) T}\right)\left(1-e^{-\left(b+\gamma_{1}+\delta \beta\right) T}\right)} \\
& -\varepsilon_{2}=m_{4} .
\end{aligned}
$$




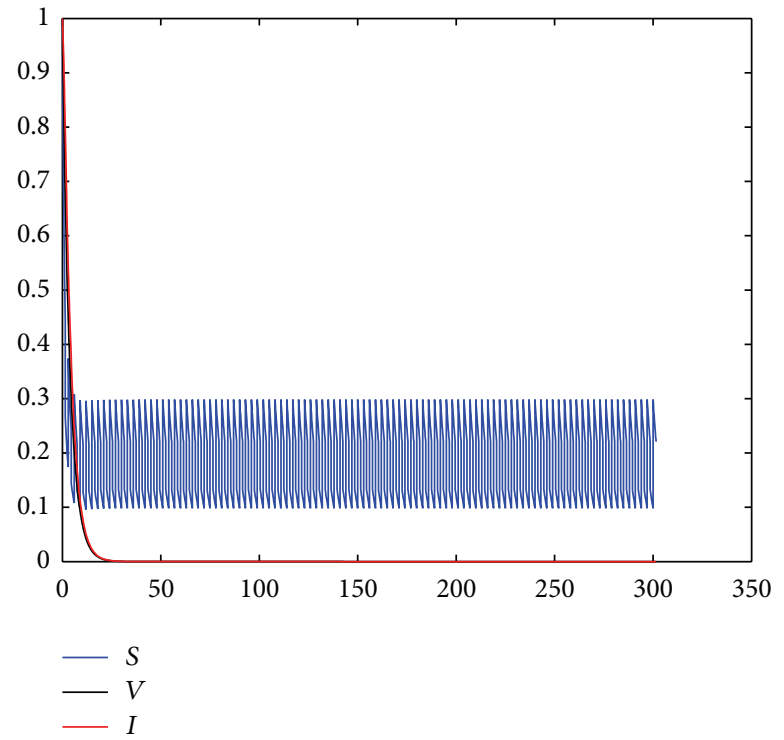

(a) Time series of $S, V$, and $I$. The disease dies out

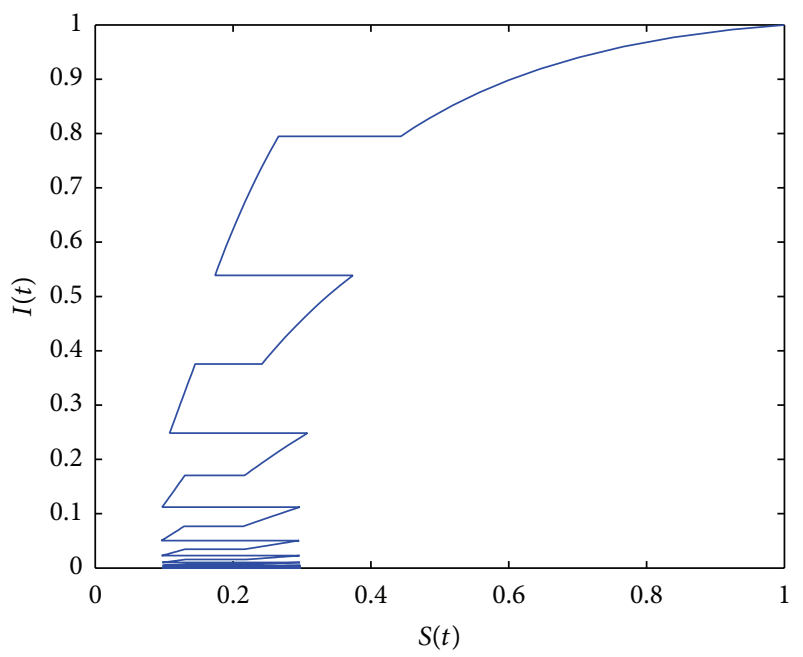

(c) Phase portrait of $S(t), I(t)$ of the system (4)

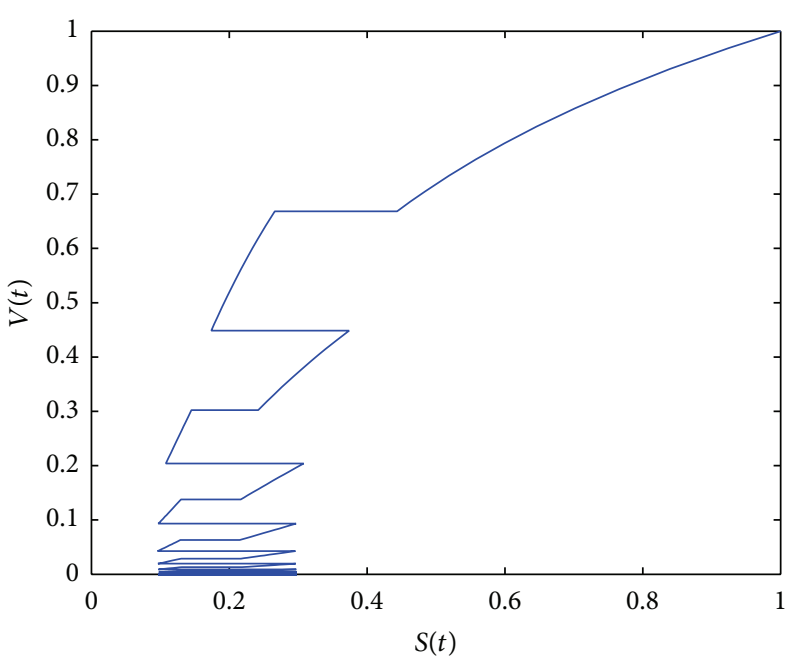

(b) Phase portrait of $S(t), V(t)$ of the system (4)

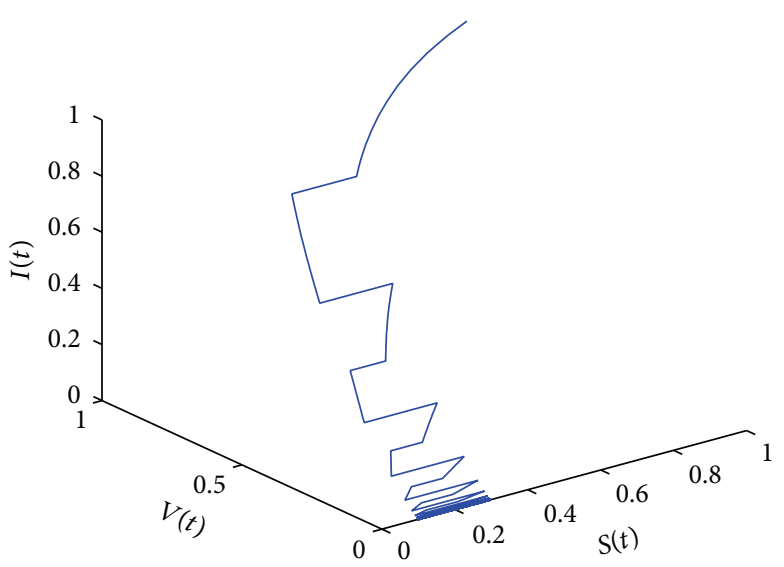

(d) Phase portrait of $S(t), V(t)$, and $I(t)$ of the system (4)

FIGURE 4: The results of numerical simulation on the threshold values $\mathscr{R}_{1}=0.0352<1$, where $p=1.5, q=1.25$.

Then for $\mathscr{D}=\left\{(S, V, I) \in R_{+}^{3} \mid S(t)+V(t)+I(t) \leq 1\right\}$, by Theorem 8 , we have

$$
m_{3} \leq S(t) \leq 1, \quad m_{1} \leq I(t) \leq 1, \quad m_{4} \leq V(t) \leq 1
$$

for $t$ large enough. Thus the system (4) is uniformly permanent.

\section{Numerical Simulations and Discussions}

Next, we carry out numerical simulations to illustrate the theoretical results obtained in the previous sections. We first set the parameters as follows: $b=0.2, \beta=0.5, \alpha=0.05$, $\gamma=0.04, \delta=0.02, \gamma_{1}=0.06, p=1.5, q=1.25, T=1.5$, $\omega=1, \tau=1, \theta=0.4$, and $\mu=1.4$. Straightforward calculation shows $\mathscr{R}_{2}=2.6155>1$. Then by Theorem 8 , the disease will be permanent (please see Figures 1(a), 1(b), $1(c)$, and $1(d))$. In order to show the effect of $\tau$, we decrease $\tau$ to 4 , and other parameters are the same with those in Figure 1, and the infection-free periodic solution of system (4) is globally attractive. This phenomenon is also seen from our theoretical analysis as in this case $\mathscr{R}_{1}=0.0339<1$ and then according to Theorem 5 , the disease will be eradicated; please see Figure 2(a).

If we keep $\tau=\omega=1$ and $\mu=1$, as the same with those in Figure 1, but increase vaccination proportion of susceptible persons $\theta$ to 0.9 , then the disease will be eradicated; see Figure 3(a). If we keep $\tau=\omega=1$ and $\theta=0.4$ and decrease $\mu$ to 0.2 , then the disease also will be eradicated; see Figure 4(a).

And if we keep $\tau=\omega=4, \mu=1$ but decrease $\theta$ to 0.1 , then the disease will be permanent; see Figure 5. If we keep $\tau=\omega=$ 


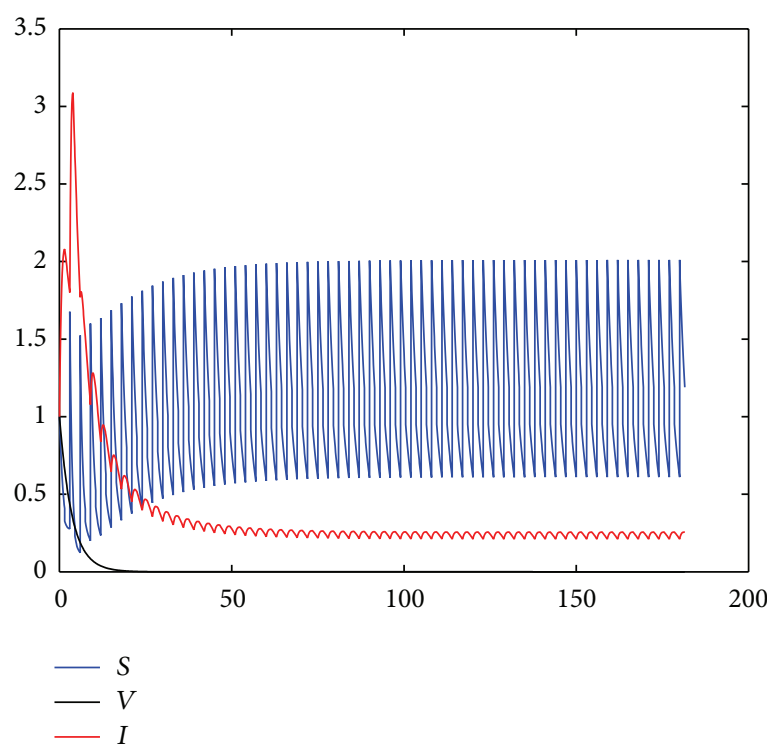

(a) Time series of $S, V$, and $I$. The disease will be permanent

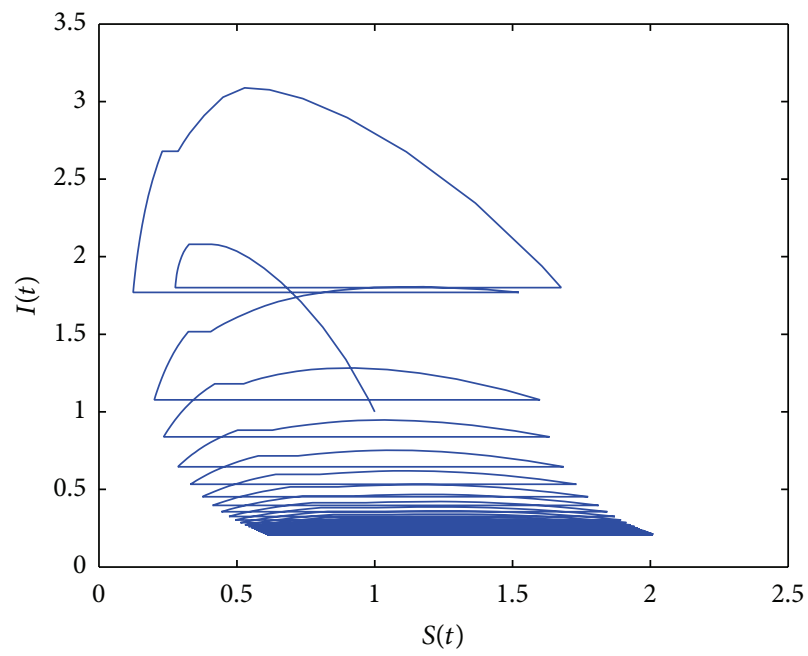

(c) Phase portrait of $S(t), I(t)$ of the system (4)

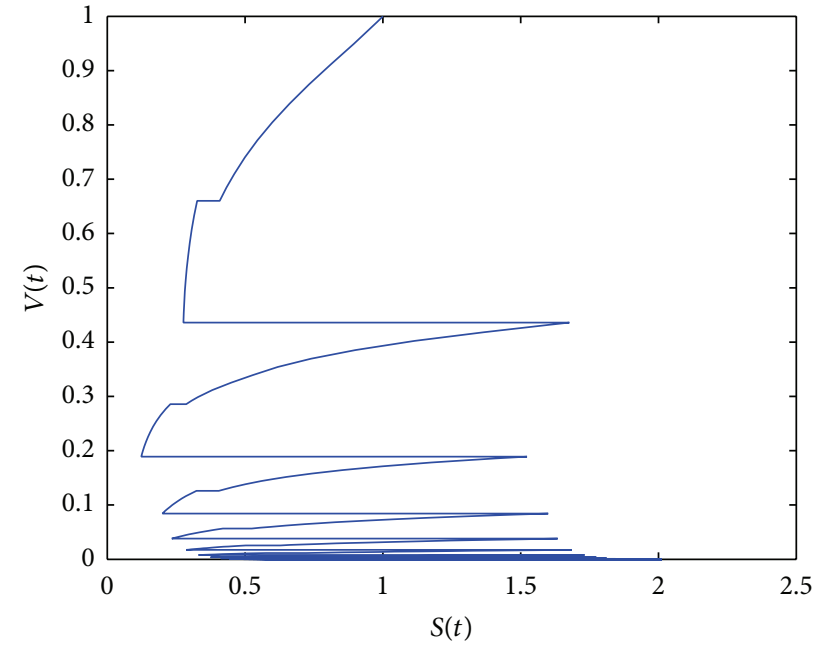

(b) Phase portrait of $S(t), V(t)$ of the system (4)

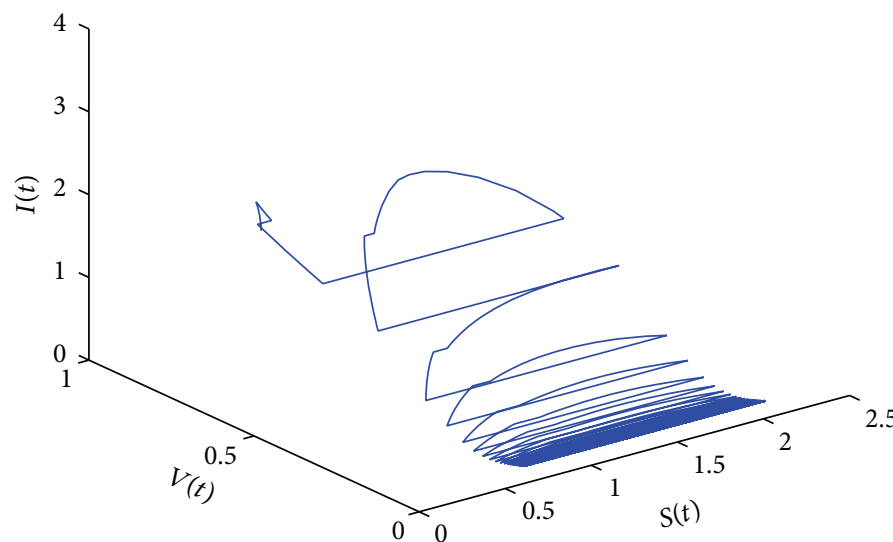

(d) Phase portrait of $S(t), V(t)$, and $I(t)$ of the system (4)

FIgURE 5: The results of numerical simulation on the threshold values $\mathscr{R}_{2}=1.1036>1$, where $p=1.5, q=1.25$.

TABLE 1

\begin{tabular}{cccccc}
\hline$\omega$ & $\tau$ & $\theta$ & $\mu$ & $\mathscr{R}_{i}$ & Status of the disease \\
\hline 1 & 1 & 0.4 & 1.4 & $\mathscr{R}_{2}=2.6155>1$ & Permanence \\
4 & 4 & 0.4 & 1.4 & $\mathscr{R}_{1}=0.0339<1$ & Eradication \\
1 & 1 & 0.9 & 1.4 & $\mathscr{R}_{1}=0.0449<1$ & Eradication \\
1 & 1 & 0.4 & 0.2 & $\mathscr{R}_{1}=0.0352<1$ & Eradication \\
4 & 4 & 0.1 & 1.4 & $\mathscr{R}_{2}=1.1036>1$ & Permanence \\
4 & 4 & 0.4 & 2 & $\mathscr{R}_{2}=2.3121>1$ & Permanence \\
\hline
\end{tabular}

4 and $\theta=0.4$ and increase $\mu$ to 2 , then the disease also will be permanent; see Figure 6. For details please see Table 1.
Lastly, we conclude our paper as follows. In this paper, we proposed an SVEIRS model, which is a new epidemic model with periodic pulse vaccination and pulse population input at two different fixed moments. Our primary result is to investigate the effect of impulsive vaccination, pulse population input, and time delays to the dynamics of population model. With the help of comparison theorems, we proved the existence of the "infection-free" periodic solution and obtained the conditions for global attractivity of the "infection-free" periodic solution and the conditions for the permanence of the system. All the theoretical results show that we believe it might be helpful in disease control: people can select appropriate vaccination rate and population input rate according to our theoretical results to control diseases. 


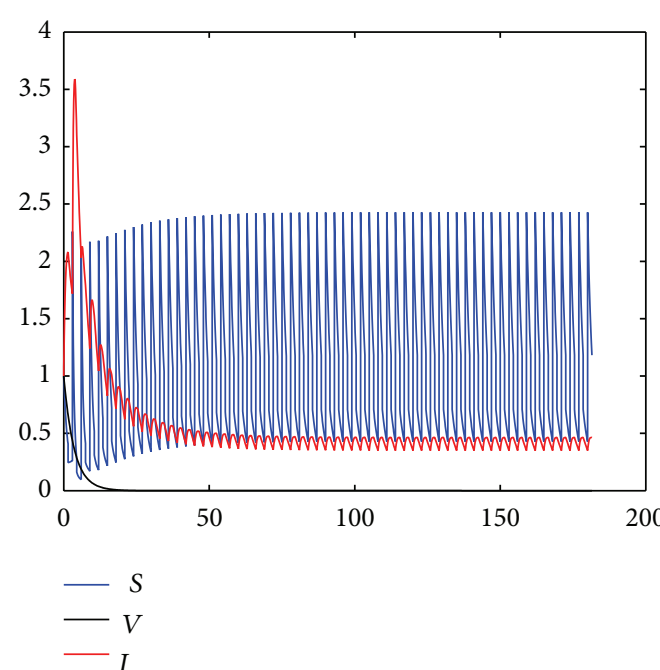

(a) Time series of $S, V$, and $I$. The disease will be permanent

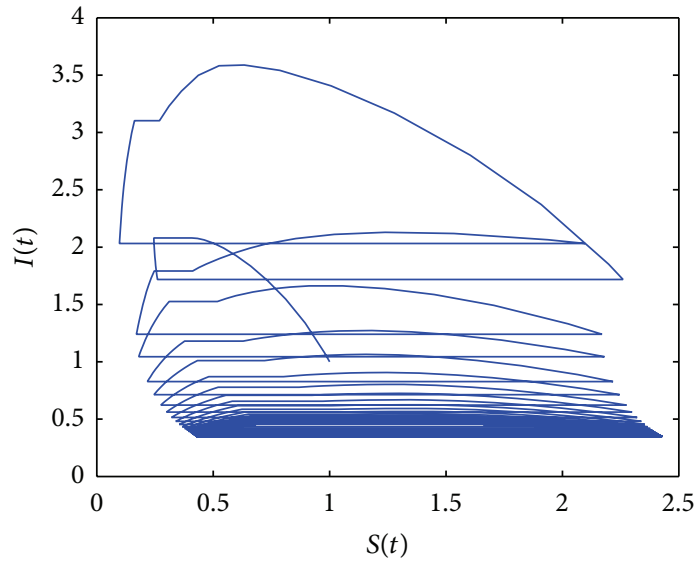

(c) Phase portrait of $S(t), I(t)$ of the system (4)

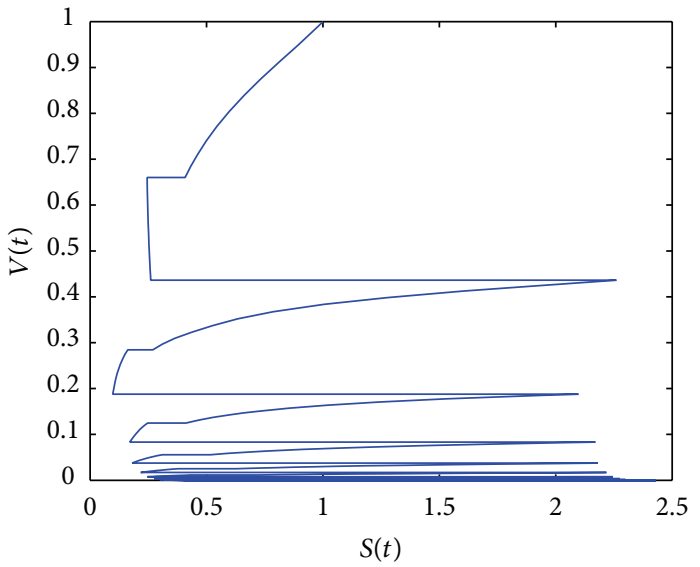

(b) Phase portrait of $S(t), V(t)$ of the system (4)

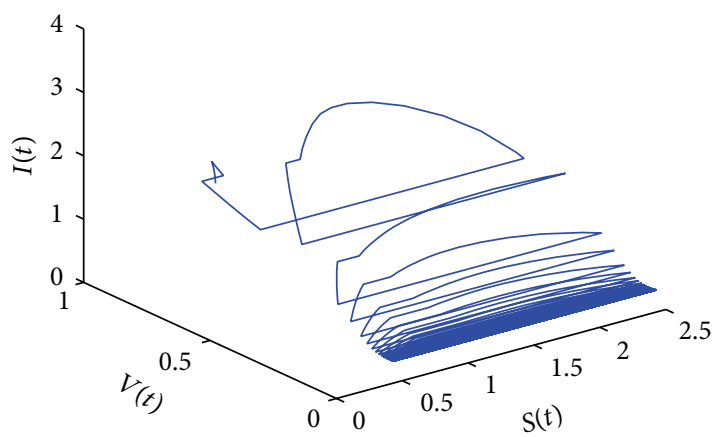

(d) Phase portrait of $S(t), V(t)$, and $I(t)$ of the system (4)

FiguRE 6: The results of numerical simulation on the threshold values $\mathscr{R}_{2}=2.3121>1$, where $p=1.5, q=1.25$.

\section{Conflict of Interests}

The authors declare that there is no conflict of interests regarding the publication of this paper.

\section{Acknowledgments}

The authors would like to thank the anonymous referees for their valuable comments and suggestions. T. Q. Zhang and X. Z. Meng are financially supported by the National Natural Science Foundation of China (no. 11371230), the Shandong Provincial Natural Science Foundation of China (no. ZR2012AM012), and the Project of Shandong Province Higher Educational Science and Technology Program of China (no. J13LI05).

\section{References}

[1] W. Piyawong, E. H. Twizell, and A. B. Gumel, "An unconditionally convergent finite-difference scheme for the SIR model,"
Applied Mathematics and Computation, vol. 146, no. 2-3, pp. 611625, 2003.

[2] A. Korobeinikov and G. C. Wake, "Lyapunov functions and global stability for SIR, SIRS, and SIS epidemiological models," Applied Mathematics Letters, vol. 15, no. 8, pp. 955-960, 2002.

[3] E. Beretta and Y. Takeuchi, "Convergence results in SIR epidemic models with varying population sizes," Nonlinear Analysis: Theory, Methods \& Applications, vol. 28, no. 12, pp. 1909-1921, 1997.

[4] E. Beretta and Y. Takeuchi, "Global stability of an SIR epidemic model with time delays," Journal of Mathematical Biology, vol. 33, no. 3, pp. 250-260, 1995.

[5] W. Ma, M. Song, and Y. Takeuchi, "Global stability of an SIR epidemic model with time delay," Applied Mathematics Letters, vol. 17, no. 10, pp. 1141-1145, 2004.

[6] Z. Jin and Z. Ma, "The stability of an SIR epidemic model with time delays," Mathematical Biosciences and Engineering, vol. 3, no. 1, pp. 101-109, 2006.

[7] J. Hui and L.-S. Chen, "Impulsive vaccination of SIR epidemic models with nonlinear incidence rates," Discrete and Continuous Dynamical Systems B, vol. 4, no. 3, pp. 595-605, 2004. 
[8] X. Meng and L. Chen, "The dynamics of a new SIR epidemic model concerning pulse vaccination strategy," Applied Mathematics and Computation, vol. 197, no. 2, pp. 582-597, 2008.

[9] G. Z. Zeng, L. S. Chen, and L. H. Sun, "Complexity of an SIR epidemic dynamics model with impulsive vaccination control," Chaos, Solitons \& Fractals, vol. 26, no. 2, pp. 495-505, 2005.

[10] A. D'Onofrio, "Pulse vaccination strategy in SIR epidemic model," Mathematical and Computer Modelling, vol. 13, pp. 1526, 2002.

[11] S. L. Yuan, Z. E. Ma, and M. A. Han, "Global stability of an SIS epidemic model with time delays," Acta Mathematica Scientia $A$, vol. 25, no. 3, pp. 349-356, 2005.

[12] S.-L. Yuan, Z.-E. Ma, and Z. Jin, "Persistence and periodic solution on a nonautonomous SIS model with delays," Acta Mathematicae Applicatae Sinica, vol. 19, no. 1, pp. 167-176, 2003.

[13] D. Kühnert, T. Stadler, T. G. Vaughan, and A. J. Drummond, "Simultaneous reconstruction of evolutionary history and epidemiological dynamics from viral sequences with the birthdeath SIR model," Journal of the Royal Society Interface, vol. 11, no. 94, Article ID 20131106, 2014.

[14] T. K. Kar and P. K. Mondal, "Global dynamics and bifurcation in delayed SIR epidemic model," Nonlinear Analysis: Real World Applications, vol. 12, no. 4, pp. 2058-2068, 2011.

[15] G. Huang and Y. Takeuchi, "Global analysis on delay epidemiological dynamic models with nonlinear incidence," Journal of Mathematical Biology, vol. 63, no. 1, pp. 125-139, 2011.

[16] C. Vargas-De-León, "On the global stability of SIS, SIR and SIRS epidemic models with standard incidence," Chaos, Solitons \& Fractals, vol. 44, no. 12, pp. 1106-1110, 2011.

[17] T. K. Kar and A. Batabyal, "Stability analysis and optimal control of an SIR epidemic model with vaccination," BioSystems, vol. 104, no. 2-3, pp. 127-135, 2011.

[18] Z. Hu, W. Ma, and S. Ruan, "Analysis of SIR epidemic models with nonlinear incidence rate and treatment," Mathematical Biosciences, vol. 238, no. 1, pp. 12-20, 2012.

[19] X. Song, Y. Jiang, and H. Wei, "Analysis of a saturation incidence SVEIRS epidemic model with pulse and two time delays," Applied Mathematics and Computation, vol. 214, no. 2, pp. 381390, 2009.

[20] T. Zhang and Z. Teng, "Global behavior and permanence of SIRS epidemic model with time delay," Nonlinear Analysis: Real World Applications, vol. 9, no. 4, pp. 1409-1424, 2008.

[21] M. Y. Li, H. L. Smith, and L. Wang, "Global dynamics an SEIR epidemic model with vertical transmission," SIAM Journal on Applied Mathematics, vol. 62, no. 1, pp. 58-69, 2001.

[22] W. Wang, "Global behavior of an SEIRS epidemic model with time delays," Applied Mathematics Letters, vol. 15, no. 4, pp. 423428, 2002.

[23] J. Zhen, Z. Ma, and M. Han, "Global stability of an SIRS epidemic model with delays," Acta Mathematica Scientia B, vol. 26, no. 2, pp. 291-306, 2006.

[24] G. Li and Z. Jin, "Global stability of a SEIR epidemic model with infectious force in latent, infected and immune period," Chaos, Solitons \& Fractals, vol. 25, no. 5, pp. 1177-1184, 2005.

[25] K. L. Cooke and P. van den Driessche, "Analysis of an SEIRS epidemic model with two delays," Journal of Mathematical Biology, vol. 35, no. 2, pp. 240-260, 1996.

[26] S. Gao, Z. Teng, and D. Xie, "The effects of pulse vaccination on SEIR model with two time delays," Applied Mathematics and Computation, vol. 201, no. 1-2, pp. 282-292, 2008.
[27] S. Gao, L. Chen, and Z. Teng, "Impulsive vaccination of an SEIRS model with time delay and varying total population size," Bulletin of Mathematical Biology, vol. 69, no. 2, pp. 731-745, 2007.

[28] X. Meng, L. Chen, and H. Cheng, "Two profitless delays for the SEIRS epidemic disease model with nonlinear incidence and pulse vaccination," Applied Mathematics and Computation, vol. 186, no. 1, pp. 516-529, 2007.

[29] T. Zhang and Z. Teng, "Pulse vaccination delayed SEIRS epidemic model with saturation incidence," Applied Mathematical Modelling, vol. 32, no. 7, pp. 1403-1416, 2008.

[30] Y. Jiang, L. Mei, and X. Song, "Global analysis of a delayed epidemic dynamical system with pulse vaccination and nonlinear incidence rate," Applied Mathematical Modelling, vol. 35, no. 10, pp. 4865-4876, 2011.

[31] K. L. Cooke, "Stability analysis for a vector disease model," The Rocky Mountain Journal of Mathematics, vol. 9, no. 1, pp. 31-42, 1979.

[32] Y. Takeuchi, W. Ma, and E. Beretta, "Global asymptotic properties of a delay SIR epidemic model with finite incubation times," Nonlinear Analysis: Theory, Methods \& Applications, vol. 42, no. 6, pp. 931-947, 2000.

[33] W.-M. Liu, H. W. Hethcote, and S. A. Levin, "Dynamical behavior of epidemiological models with nonlinear incidence rates," Journal of Mathematical Biology, vol. 25, no. 4, pp. 359$380,1987$.

[34] W.-M. Liu, S. A. Levin, and Y. Iwasa, "Influence of nonlinear incidence rates upon the behavior of SIRS epidemiological models," Journal of Mathematical Biology, vol. 23, no. 2, pp. 187240, 1986.

[35] X. Meng, L. Chen, and B. Wu, "A delay SIR epidemic model with pulse vaccination and incubation times," Nonlinear Analysis: Real World Applications, vol. 11, no. 1, pp. 88-98, 2010.

[36] F. N. M. Al-Showaikh and E. H. Twizell, "One-dimensional measles dynamics," Applied Mathematics and Computation, vol. 152, no. 1, pp. 169-194, 2004.

[37] D. Greenhalgh, "Hopf bifurcation in epidemic models with a latent period and nonpermanent immunity," Mathematical and Computer Modelling, vol. 25, no. 2, pp. 85-107, 1997.

[38] A. d'Onofrio, "Mixed pulse vaccination strategy in epidemic model with realistically distributed infectious and latent times," Applied Mathematics and Computation, vol. 151, no. 1, pp. 181187, 2004.

[39] V. Lakshmikantham, D. D. Baĭnov, and P. S. Simeonov, Theory of Impulsive Differential Equations, vol. 6 of Series in Modern Applied Mathematics, World Scientific, Singapore, 1989.

[40] D. Bainov and P. Simeonov, System with Impulsive Effect: Stability, Theory and Applications, John Wiley \& Sons, New York, NY, USA, 1989.

[41] Y. Kuang, Delay Differential Equations with Applications in Population Dynamics, vol. 191 of Mathematics in Science and Engineering, Academic Press, San Diego, Calif, USA, 1993.

[42] T. Zhang, X. Meng, and Y. Song, "The dynamics of a highdimensional delayed pest management model with impulsive pesticide input and harvesting prey at different fixed moments," Nonlinear Dynamics, vol. 64, no. 1-2, pp. 1-12, 2011. 


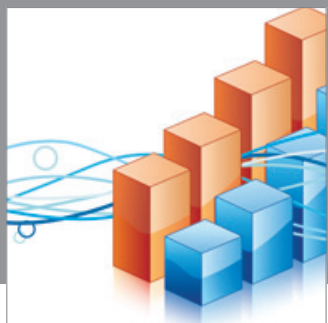

Advances in

Operations Research

mansans

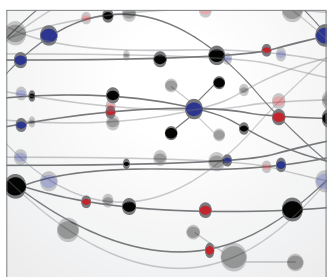

The Scientific World Journal
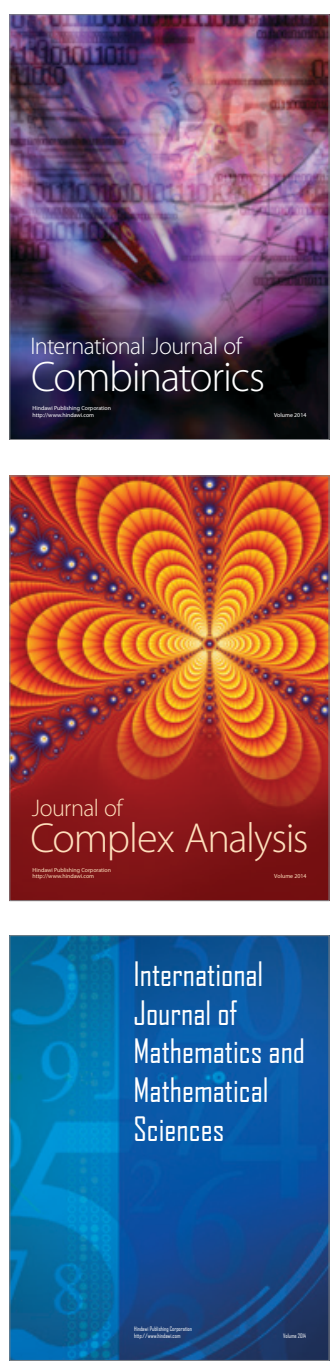
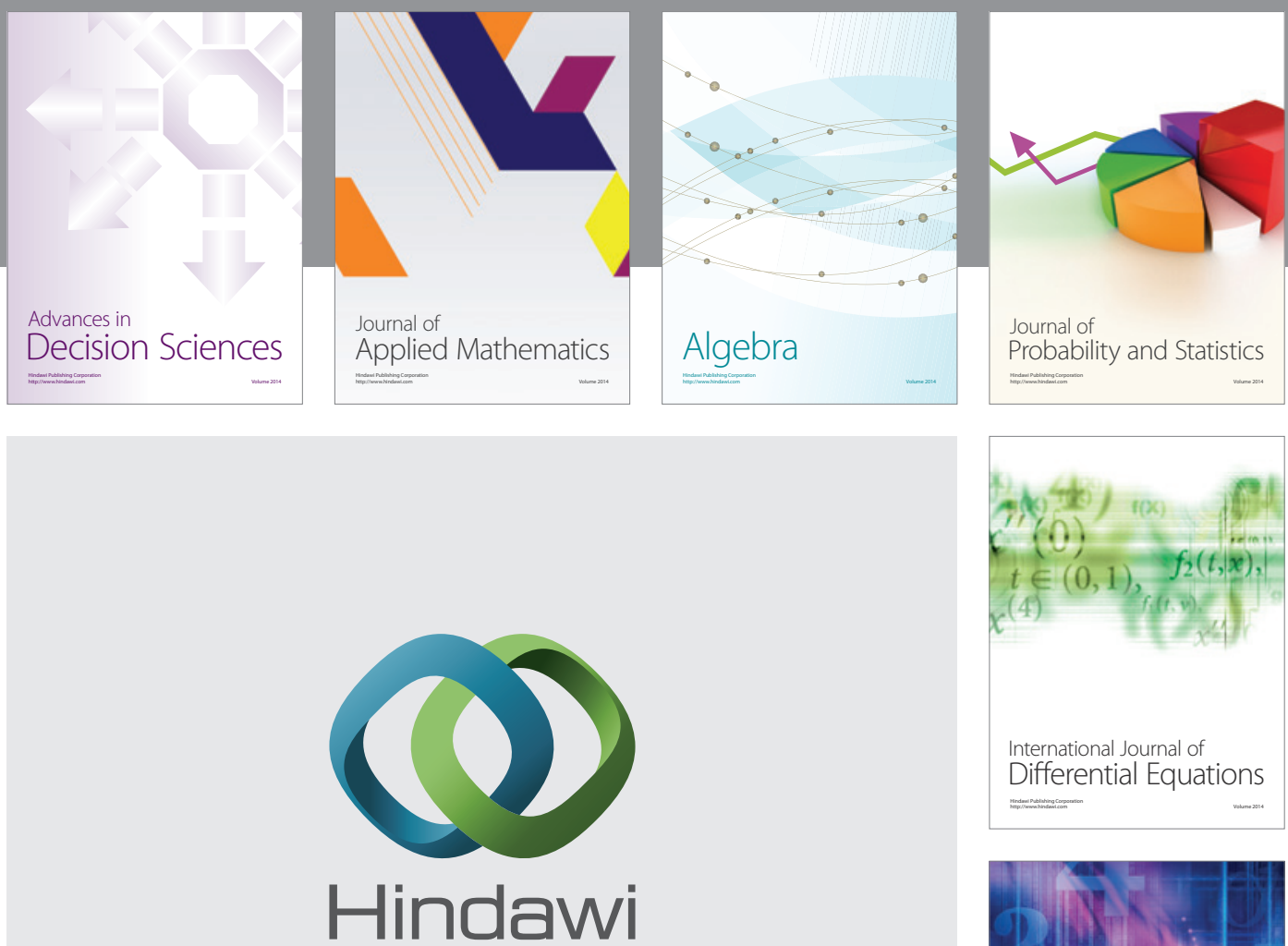

Submit your manuscripts at http://www.hindawi.com
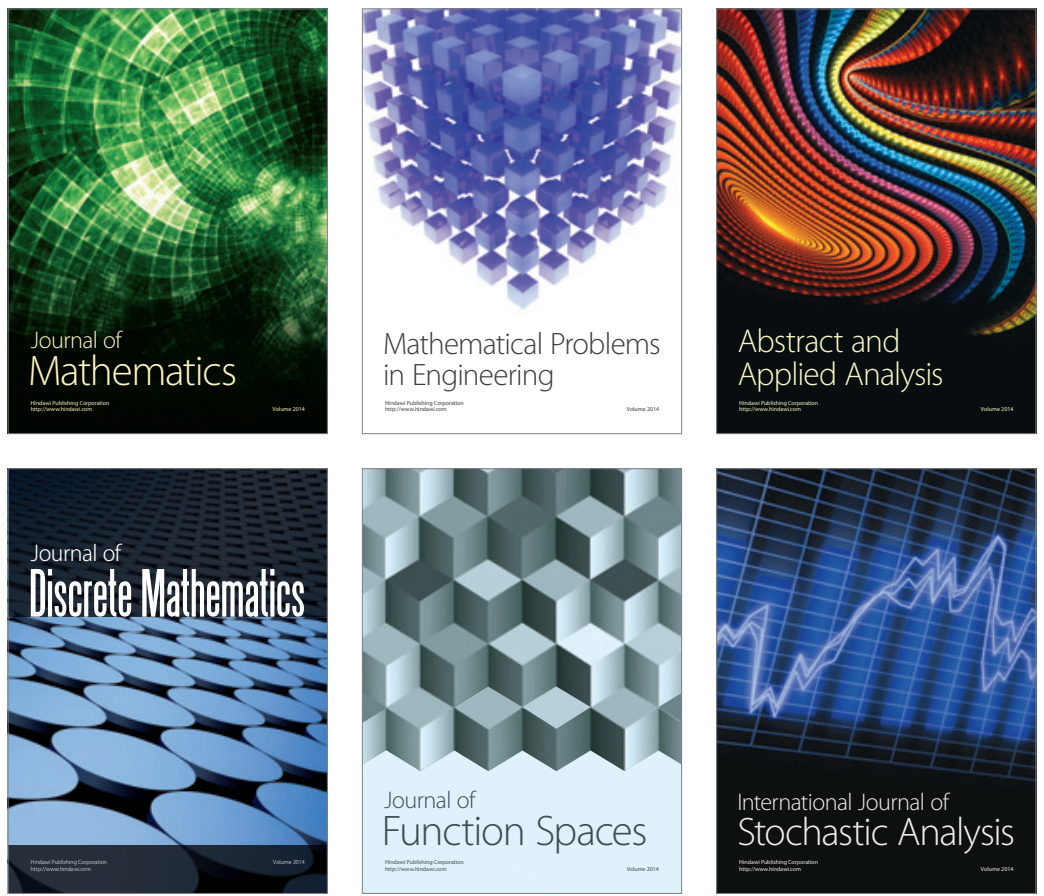

Journal of

Function Spaces

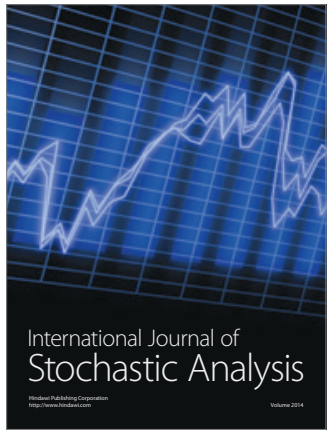

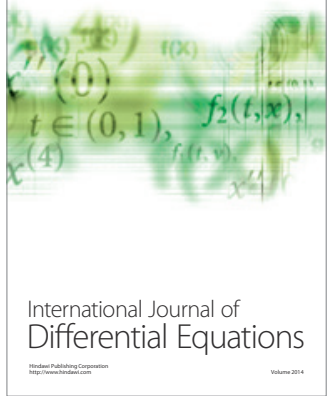
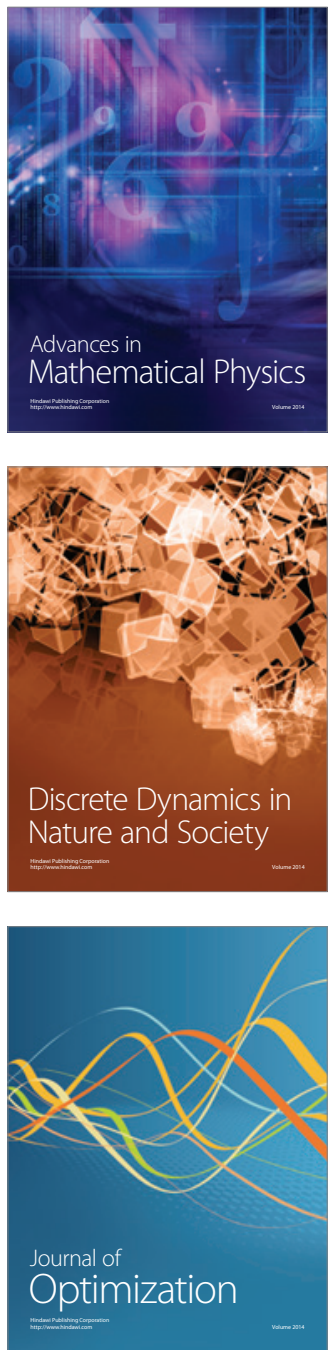